\title{
ON A COMPUTATIONAL ALGORITHM FOR A CLASS OF OPTIMAL CONTROL PROBLEMS INVOLVING DISCRETE TIME DELAYED ARGUMENTS
}

\author{
J. M. MURRAY and K. L. TEO
}

(Received 22 December 1977)

(Revised 23 May 1978)

\begin{abstract}
In this paper, a computational algorithm for solving a class of optimal control problems involving discrete time-delayed arguments is presented. By way of example, a simple model of a production firm is devised for which the algorithm is used to solve a decision-making problem.
\end{abstract}

\section{Introduction}

Recently, computational algorithms for solving optimal control problems of ordinary dynamical systems using strong variations in control have been reported in [6]. In their investigation, a control $\hat{u}$ which maximizes a Hamiltonian function is calculated and the new control is formed according to the formula

$$
u_{\alpha}(t)= \begin{cases}\hat{u}(t) & \text { for all } t \in I_{\alpha u} \\ u(t) & \text { otherwise, }\end{cases}
$$

where $I_{\alpha u}$ is a subset of the time interval, having a total length $\alpha$. Different methods have been suggested in [8] for constructing the set $I_{\alpha u}$ so that the corresponding control $u_{\alpha}$ will improve the objective functional.

In this paper, results leading up to a computational algorithm for solving optimal control problems that include time delays are devised. However, no attempt is made to prove the convergence of this algorithm, as is done in [6] for the ordinary dynamical system. Note that the delays, which are taken to be 
discrete, are allowed to occur in both the state and control variables. The initial function $\phi$ is presumed given and absolutely continuous.

In Section 4, two theorems are presented which motivate the algorithm to be given in Section 5. The first of these, Theorem 4.4, demonstrates that if $u$ is not an extremal control then a new control $u_{\alpha}$ can be constructed so that the corresponding objective functional will be improved. However, the main disadvantage of this theorem is that it requires exact maximization of the Hamiltonian for all $t \in[0, T]$. In order to achieve some sort of practicality in the algorithm, this stringent requirement has to be relaxed. Theorem 4.5, an extension of Theorem 4.4, which maintains much the same hypotheses, requires only approximate maximization of the Hamiltonian at one point in $[0, T]$. On the basis of this theorem, a computational algorithm is formulated in Section 5. It should be noted that this paper was motivated by the results given in [2], [6] and [8].

For illustration, this algorithm is used to solve a production-inventory model. In this model, the objective is to derive the best possible production and advertising policies with respect to a desired profit functional, where the controls for both production and advertising incorporate delays.

\section{Statement of the problem, basic definitions and assumptions}

Consider the following delay-differential equation on the fixed time interval $(0, T]$

$$
\dot{x}(t)=\sum_{j=0}^{8} f^{j}\left(t-h_{j}, x\left(t-h_{j}\right), u\left(t-h_{j}\right)\right),
$$

where $x \triangleq\left[x_{1}, \ldots, x_{n}\right]^{\mathrm{T}} \in R^{n}, u \triangleq\left[u_{1}, \ldots, u_{r}\right]^{\mathrm{T}} \in R^{r}$ are, respectively, the state and control vectors, $f^{j} \triangleq\left[f_{1}^{j}, \ldots, f_{n}^{j}\right]^{\mathrm{T}} \in R^{n}(j=0, \ldots, s)$, and the superscript $\mathrm{T}$ denotes the transpose. The $h_{k}$ are the time-delays, ordered so that

$$
0=h_{0}<h_{1}<\ldots<h_{s}<T ; s<\infty .
$$

The initial function for equation (1) is

$$
x(t)=\phi(t), \quad t \in\left[-h_{B}, 0\right],
$$

where $\phi \triangleq\left[\phi_{1}, \ldots, \phi_{n}\right]^{\mathrm{T}}$ is a given, absolutely continuous function on $\left[-h_{s}, 0\right]$ with values in $R^{n}$.

Let $D$ be the class of all admissible controls defined by

$D \triangleq\left\{u: u\right.$ is a function from $\left[-h_{s}, T\right]$ into $U$, piecewise continuous on $[0, T]$ and with $u(t)=\beta(t)$ on $\left.\left[-h_{s}, 0\right)\right\}$ where $\beta$ is a given piecewise continuous function on $\left[-h_{s}, 0\right)$ with values in $U$, and $U$ is a compact and convex subset of $\boldsymbol{R}^{\boldsymbol{r}}$. 
For each $u \in D$, let $x(u)$ denote the corresponding solution of the system (1)-(2). We may now state our problem, denoted by $P$, as: subject to the dynamic constraint (1) with the initial condition (2), find a control $u \in D$ that will minimize the cost functional $J$ defined by

$$
J(u)=\int_{0}^{T} \sum_{j=0}^{s} f_{0}^{j}\left(t-h_{j}, x(u)\left(t-h_{j}\right), u\left(t-h_{j}\right)\right) d t
$$

where for each $j \in\{0,1, \ldots, s\}, f_{0}^{j}$ is a real-valued function.

Now let us introduce some useful notation. For any $z \in R^{n}$ let

$$
\|z\| \triangleq\left(\sum_{i=1}^{n}\left(z_{i}\right)^{2}\right)^{i}
$$

For any function $g: R^{n} \rightarrow R^{n}$, let

$$
\frac{\partial g}{\partial x} \triangleq\left(\begin{array}{ccc}
\frac{\partial g_{1}}{\partial x_{1}} & \cdots & \frac{\partial g_{n}}{\partial x_{1}} \\
\vdots & & \vdots \\
\frac{\partial g_{1}}{\partial x_{n}} & \cdots & \frac{\partial g_{n}}{\partial x_{n}}
\end{array}\right) .
$$

Throughout this paper, we need the following assumptions which will be referred to collectively as (A)

(i)

$$
\begin{aligned}
& f^{j}: R^{1} \times R^{n} \times R^{r} \rightarrow R^{n} \quad(j=0, \ldots, s), \\
& f_{0}^{j}: R^{1} \times R^{n} \times R^{r} \rightarrow R^{1} \quad(j=0, \ldots, s) .
\end{aligned}
$$

(ii) $f_{i}^{j}$ and $\partial f_{i}^{j} / \partial x_{k}(j=0, \ldots, s ; i=0,1, \ldots, n ; k=1, \ldots, n)$ are piecewise continuous on $[0, T]$ for all $(x, u) \in R^{n} \times U$ and continuous on $R^{n} \times U$ for each $t \in[0, T]$.

(iii) For any given compact set $\Omega \subset R^{n}$, there exists a positive constant $m$ so that

and

$$
\left\|f^{j}(t, x, v)\right\| \leqslant m(1+\|x\|)
$$

$$
\left\|f_{0}^{j}(t, x, v)\right\| \leqslant m(1+\|x\|)
$$

for all $j=0, \ldots, s$ and for all $(t, x, v) \in[0, T] \times \Omega \times U$.

In the results to follow, we need

DeFINITION 2.1. For each $u \in D$, the absolutely continuous function

$$
\psi(u) \triangleq\left[\psi_{0}(u), \psi_{1}(u), \ldots, \psi_{n}(u)\right]^{\mathrm{T}}:[0, T] \rightarrow R^{n+1}
$$

is the solution of

$$
\left.\begin{array}{l}
\psi_{i}(t)=-\sum_{k=0}^{n} \sum_{j=0}^{s} \frac{\partial f_{k}^{j}(t, x(t), u(t))}{\partial x_{i}} \psi_{k}\left(t+h_{j}\right) \quad(i=1, \ldots, n), \\
\psi_{0}(t)=-1
\end{array}\right\}
$$


with the final condition

and

$$
\left.\begin{array}{l}
\psi_{i}(t)=0 \quad \text { for all } t \geqslant T \quad(i=1, \ldots, n) \\
\psi_{0}(t)=0 \text { for all } t>T .
\end{array}\right\}
$$

DEFINITION 2.2. Using the abbreviation $\Psi(t)$ for $\psi\left(t+h_{0}\right), \psi\left(t+h_{1}\right), \ldots, \psi\left(t+h_{s}\right)$ we define the Hamiltonian $H:[0, T] \times R^{n} \times U \times R^{(n+1)(s+1)} \rightarrow R^{1}$ to be

$$
H(t, x, v, \Psi) \triangleq \sum_{k=0}^{n} \sum_{j=0}^{s} f_{k}^{j}(t, x, v) \psi_{k}\left(t+h_{j}\right)
$$

THEOREM 2.3. For each control $u \in D$, there exists a unique absolutely continuous vector-valued function $x$ which satisfies the system (1)-(2).

Proof. If we subdivide the interval $[0, T]$ into $\left[0, h_{1}\right],\left[v h_{1},(v+1) h_{1}\right](v=1, \ldots, k)$, $\left[(k+1) h_{1}, T\right]$, then finding the unique solution of the system (1)-(2) is the same as finding unique solutions of the system (1) on each of these subintervals successively with the appropriate boundary conditions. Thus, the proof follows from repeated applications of Theorem 1 of [4].

We shall now impose certain conditions $\left(A_{H}\right)$ on the Hamiltonian:

(i) for every $u \in D$, the function

$$
H:(t, v) \rightarrow H(t, x(u)(t), v, \Psi(u)(t))
$$

is piecewise continuous on $[0, T]$ for each $v \in U$ and continuous on $U$ for each $t \in[0, T]$; and

(ii) for every $u \in D$, there is a partition of $[0, T]$ into intervals $I^{i}(u)(i \in Q(u))$, such that whenever $t$ is in the interior of any $I^{i}, H(t, x(u)(t), v, \Psi(u)(t))$ is continuous in $(t, v)$ where $v \in U$, and has a unique maximum as a function of $v \in U$.

\section{Certain preparatory results}

In order to devise a computational algorithm for approximating problem $P$, we need certain preparatory results, to be presented in this Section.

REMARK 3.1. For each $u \in D$, the function

$$
V_{u}:[0, T] \rightarrow U
$$

is such that for any $t$ in the interior of any of the $I^{i}(u), V_{u}$ maximizes $H(t, x(u)(t), \cdot, \Psi(u)(t))$ and for all other $t \in[0, T], V_{u}(t)=u(t)$. 
Note that under the assumptions $\left(A_{H}\right), V_{u}$ has the property that if $t$ is in the interior of any of the $I^{i}(u)$ then

$$
H\left(t, x(u)(t), V_{u}(t), \Psi(u)(t)\right) \geqslant H(t, x(u)(t), v, \Psi(u)(t))
$$

for all $v \in U$, and the equality holds if and only if $v=V_{u}(t)$. Thus, in particular

$$
H\left(t, x(u)(t), V_{u}(t), \Psi(u)(t)\right)>H(t, x(u)(t), u(t), \Psi(u)(t))
$$

whenever $u(t) \neq V_{u}(t)$.

Theorem 3.2. Suppose that the assumptions $(A)$ and $\left(A_{B}\right)$ are satisfied. Then, for each $u \in D, V_{u}$ is continuous in the interior of any of the intervals $I^{i}(u), i \in Q(u)$, where $I^{i}(u), i \in Q(u)$, are as defined before. Further, $V_{u}(t) \in U$ for all $t \in[0, T]$.

Proof. The second statement of the theorem follows as an immediate consequence of the definition of $V_{u}$.

The proof of the first statement is given below.

Let $u \in D$ and let $t^{*} \in \bigcup_{i \in Q(u)}\left(I^{i}(u)\right)^{0}$ where $\left(I^{i}(u)\right)^{0}$ denotes the interior of the interval $I^{i}(u)$. Now suppose that $V_{u}$ is not continuous at $t^{*}$. Then, there exists an $\varepsilon>0$ and a sequence of points $\left\{t_{k}\right\}_{k=1}^{\infty} \subset[0, T]$ such that

$$
\left|t_{k}-t *\right|<1 / k
$$

but

for all integers $k \geqslant 1$.

$$
\left|V_{u}\left(t_{k}\right)-V_{u}\left(t^{*}\right)\right|>\varepsilon
$$

However, $\left\{V_{u}\left(t_{k}\right)\right\}_{k=1}^{\infty}$ is a sequence in $U$ and $U$ is a compact subset of $R^{r}$. Thus, there exists a subsequence of $\left\{V_{u}\left(t_{k}\right)\right\}_{k=1}^{\infty}$ which is denoted by the original sequence, such that

$$
\lim _{k \rightarrow \infty} V_{u}\left(t_{k}\right)=V^{* *}
$$

Now let

$$
V^{*} \triangleq V_{u}\left(t^{*}\right)
$$

In view of (8), (10) and (12), we deduce that

$$
H\left(t_{k}, x(u)\left(t_{k}\right), V_{u}\left(t_{k}\right), \Psi(u)\left(t_{k}\right)\right)>H\left(t_{k}, x(u)\left(t_{k}\right), V^{*}, \Psi(u)\left(t_{k}\right)\right) .
$$

By condition (ii) of assumptions $\left(A_{H}\right)$, we note that $H(\cdot, x(u)(\cdot), \cdot, \Psi(u)(\cdot))$ is continuous on any of the sets $\left(I^{i}(u)\right)^{0} \times U, i \in Q(u)$. In particular, this function is continuous at $t=t^{*}$ and $v=V^{* *}$.

Thus, by letting $k \rightarrow \infty$ in the inequality (13) we obtain from (9) and (11) that

$$
H\left(t^{*}, x(u)\left(t^{*}\right), V^{* *}, \Psi(u)\left(t^{*}\right)\right) \geqslant H\left(t^{*}, x(u)\left(t^{*}\right), V^{*}, \Psi(u)\left(t^{*}\right)\right) .
$$


On the other hand, we deduce from (9) and (10) that for a sufficiently large positive integer $k$

Consequently,

$$
\begin{aligned}
\varepsilon & <\left|V_{u}\left(t_{k}\right)-V^{*}\right| \\
& \leqslant\left|V_{u}\left(t_{k}\right)-V^{* *}\right|+\left|V^{* *}-V^{*}\right| \\
& \leqslant \frac{1}{2} \varepsilon+\left|V^{* *}-V^{*}\right|
\end{aligned}
$$

$$
0<\frac{i}{2} \varepsilon<\left|V^{* *}-V^{*}\right|
$$

This, in turn, implies that

$$
V^{* *} \neq V^{*}
$$

Therefore, it follows from (8) that

$$
H\left(t^{*}, x(u)\left(t^{*}\right), V^{*}, \Psi^{*}(u)\left(t^{*}\right)\right)>H\left(t^{*}, x(u)\left(t^{*}\right), V^{* *}, \Psi(u)\left(t^{*}\right)\right) \text {. }
$$

This contradicts inequality (14). Thus, $V_{u}$ must be continuous at $t^{*}$. Since $t^{*}$ is an arbitrary point in $\bigcup_{i \in Q(u)}\left(I^{i}(u)\right)^{0}$, the first statement of the theorem is established. This completes the proof.

RemarK 3.3. Theorem 3.2 implies that if $u \in D$ then $V_{u} \in D$. In the sequel, we need

THEOREM 3.4. Consider the optimal control problem P. Suppose that the assumptions $(A)$ and $\left(A_{H}\right)$ are satisfied and that $u^{*} \in D$ is an optimal control. Then

$$
\begin{aligned}
\int_{0}^{T} H\left(t, x\left(u^{*}\right)(t), u^{*}(t), \Psi\left(u^{*}\right)(t)\right) d t & \\
= & \max \left\{\int_{0}^{T} H\left(t, x\left(u^{*}\right)(t), u(t), \Psi\left(u^{*}\right)(t)\right) d t: u \in D\right\} .
\end{aligned}
$$

The proof of Theorem 3.4 will be postponed until after the proof of Theorem 4.4. With reference to Theorem 3.4, we have

Definition 3.5. A control $u^{*} \in D$ is said to be an extremal control if it satisfies condition (19) of Theorem 3.4 .

REMARK 3.6. Let $\Theta^{\prime}(u)$ denote the points of discontinuity of the functions

$$
\begin{aligned}
f_{i}^{j}(\cdot, x(u)(\cdot), u(\cdot)) \text { and } \frac{\partial f_{i}^{j}(\cdot, x(u)(\cdot), u(\cdot))}{\partial x_{k}} \\
\quad(i=0,1, \ldots, n ; k=1, \ldots, n ; j=0,1, \ldots, s) .
\end{aligned}
$$


Now let $\Theta(u)$ consist of all the $\theta^{\prime} \in \Theta^{\prime}(u)$ and the points $\theta^{\prime}+h_{j}, j=1, \ldots, s$, and the endpoints of the intervals $I^{i}(u), i \in Q(u)$. In the proof of Theorem 4.4, the control is perturbed on an interval $\left[t_{c}-\varepsilon l_{1}, t_{c}+\varepsilon l_{2}\right]$ which must not include $\theta^{\prime}+h_{j}$, $j=0,1, \ldots, s$, for any $\theta^{\prime} \in \Theta^{\prime}(u)$.

Remark 3.7. For future convenience, the function $H_{\max }$ is defined as

$$
H_{\max }(t, x(u)(t), \Psi(u)(t))=\max _{v \in U} H(t, x(u)(t), v, \Psi(u)(t)) .
$$

REMARK 3.8. When a piecewise continuous function $\gamma$, having discontinuities at $a$ and $b$, is considered on the interval $[a, b], \gamma(a)$ shall mean $\gamma(a+0)$ and $\gamma(b)$ shall mean $\gamma(b-0)$.

\section{Motivation for the algorithm}

In this Section we present two theorems which motivate the algorithm in Section 5. The algorithm presented there is an implementable algorithm, as defined in [7], and is based on the results of Theorem 4.5. If a conceptual algorithm were desired it would be modelled on the results of Theorem 4.4.

Theorem 4.4 will prove that if we are given a control $u^{\mathbf{1}} \in D$ that is not an extremal control, then a new control $u^{2} \in D$ can be constructed so that the corresponding objective functional will be improved.

Let us introduce a new variable by defining

$$
x_{0}(u)(t)=\int_{0}^{t} \sum_{j=0}^{s} f_{0}^{j}\left(\tau-h_{j}, x(u)\left(\tau-h_{j}\right), u\left(\tau-h_{j}\right)\right) d \tau .
$$

Then, we have

$$
\left.\begin{array}{l}
\dot{x}_{0}(u)(t)=\sum_{j=0}^{s} f_{0}^{j}\left(t-h_{j}, x(u)\left(t-h_{j}\right), u\left(t-h_{j}\right)\right), \quad t \in(0, T] \\
x_{0}(u)(t)=0, \quad t \in\left[-h_{s}, 0\right] .
\end{array}\right\}
$$

If, in the following Remark, we divide the interval $[0, T]$ into intervals of length, at most, $h_{1}$, then the differential equations

$$
\dot{x}_{i}(t)=\sum_{j=0}^{s} f_{i}^{s}\left(t-h_{j}, x\left(t-h_{j}\right), u\left(t-h_{j}\right)\right), \quad i=0,1, \ldots, n,
$$

can be treated as differential equations without delays since all the delayed terms will occur in previous intervals and therefore will have already been calculated. 
REMARK 4.1. In this Remark we derive the variational equations for the state, when the control is perturbed.

For $u^{1} \in D$, let $\varepsilon>0$ be such that no $\theta \in \Theta\left(u^{1}\right)$ is contained in the interval $\left[t_{c}-\varepsilon l_{1}, t_{c}+\varepsilon l_{2}\right]$ and such that no $\left[t_{c}-\varepsilon l_{1}+h_{j}, t_{c}+\varepsilon l_{2}+h_{j}\right](j=0,1, \ldots, s)$ overlap, where $t_{c} \in[0, T]$ is chosen such that $V_{u^{1}}\left(t_{c}\right) \neq u^{1}\left(t_{c}\right)$. Consider the following perturbation of the control $u^{1}$ : let

and

$$
\Omega_{e}=\left[t_{c}-\varepsilon l_{1}, t_{c}+\varepsilon l_{2}\right]
$$

$$
u_{e}^{1}(t)= \begin{cases}V_{u^{1}}(t) & \text { if } t \in \Omega_{e}, \\ u^{1}(t) & \text { otherwise, }\end{cases}
$$

where $l_{1}=0$ if $t_{c}=0$ or if $t_{c} \in \Theta\left(u^{1}\right)$ and $l_{2}>0$, and $l_{1}$ is a positive constant elsewhere; and $l_{2}=0$ if $t_{c}=T$ or if $t_{c} \in \Theta\left(u^{1}\right)$ and $l_{1}>0$, and $l_{2}$ is a positive constant elsewhere. Here $\Theta\left(u^{1}\right)$ is as defined in Remark 3.6.

Combining equations (21) and (1) we have for $t \in(0, T]$

$$
\dot{x}_{i}(u)(t)=\sum_{j=0}^{s} f_{i}^{j}\left(t-h_{j}, x(u)\left(t-h_{j}\right), u\left(t-h_{j}\right)\right), \quad i=0,1, \ldots, n,
$$

with the initial conditions for $t \in\left[-h_{s}, 0\right]$

$$
\left\{\begin{array}{l}
x_{i}(u)(t)=\phi_{i}(t), \quad i=1, \ldots, n \\
x_{0}(u)(t)=0
\end{array}\right.
$$

Consider system (23) on $\left[t_{c}-\varepsilon l_{1}, t_{c}+\varepsilon l_{2}\right]$. By its construction, this interval does not contain any $\theta \in \Theta\left(u^{1}\right)$ and hence the right-hand side of (23) is continuous for $u=u^{1}$. By Theorem 3.2, it is also continuous in this interval when $u=u_{s}^{1}$. Therefore it can be easily shown that

where

$$
x_{i}\left(u_{\varepsilon}^{1}\right)\left(t_{c}+\varepsilon l_{2}\right)=x_{i}\left(u^{1}\right)\left(t_{c}+\varepsilon l_{2}\right)+\varepsilon \xi_{i}\left(u^{1}\right)\left(t_{c}+\varepsilon l_{2}\right)+o(\varepsilon),
$$

$$
\begin{aligned}
\xi_{i}\left(u^{1}\right)\left(t_{c}+\varepsilon l_{2}\right)=\left(l_{1}+l_{2}\right) \cdot\left[f_{i}^{0}\left(t_{c}+\varepsilon l_{2}, x\left(u^{1}\right)\left(t_{c}+\varepsilon l_{2}\right), V_{u^{1}}\left(t_{c}+\varepsilon l_{2}\right)\right)\right. & \\
& \left.-f_{i}^{0}\left(t_{c}+\varepsilon l_{2}, x\left(u^{1}\right)\left(t_{c}+\varepsilon l_{2}\right), u^{1}\left(t_{c}+\varepsilon l_{2}\right)\right)\right], \quad i=0,1, \ldots, n,
\end{aligned}
$$

and $o(\varepsilon)$ is such that $\lim _{\varepsilon \downarrow 0}\|o(\varepsilon)\| / \varepsilon=0$.

By the definition of $u_{\varepsilon}^{1}$ given in (22) we observe that $u_{\varepsilon}^{1}(t)=u^{1}(t)$ for $t \in\left[t_{c}+\varepsilon l_{2}, t_{c}-\varepsilon l_{1}+h_{1}\right]$. Therefore the difference between the trajectories $x\left(u^{1}\right)(t)$ and $x\left(u_{\varepsilon}^{1}\right)(t)$ on this interval will result only from the differing initial conditions for the differential equation (23). The perturbation of the initial conditions is given in equation (24). Thus, we may use [1, Lemma 4.3 with $r=1, \delta t_{0}=\delta t_{1}=0$, $\delta F(t, y)=0$ and $g(t, y, \alpha, \varepsilon)=0$, pp. 258-259] to obtain

$$
x_{i}\left(u_{\varepsilon}^{1}\right)(t)=x_{i}\left(u^{1}\right)(t)+\varepsilon \delta x_{i}\left(u^{1}\right)(t)+o(\varepsilon)
$$


for $t \in\left[t_{c}+\varepsilon l_{2}, t_{c}-\varepsilon l_{1}+h_{1}\right]$, where

$$
\delta \dot{x}_{i}\left(u^{1}\right)(t)=\sum_{k=0}^{n} \sum_{j=0}^{s} \frac{\partial f_{i}^{j}\left(t-h_{j}, x\left(u^{1}\right)\left(t-h_{j}\right), u^{1}\left(t-h_{j}\right)\right)}{\partial x_{k}} \delta x_{k}\left(u^{1}\right)\left(t-h_{j}\right)
$$

for $t \in\left(t_{c}+\varepsilon l_{2}, t_{c}-\varepsilon l_{1}+h_{1}\right]$, with the initial conditions

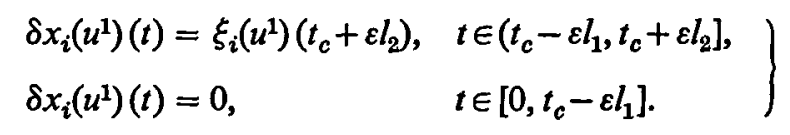

This procedure is repeated for intervals of length $h_{1}$, for example

$$
\left[t_{c}-\varepsilon l_{1}+h_{1}, t_{c}-\varepsilon l_{1}+2 h_{1}\right]
$$

or until one of the points $t_{c}-\varepsilon l_{1}+h_{j}(j=2, \ldots, s)$, is reached, for example $\left[t_{c}-\varepsilon l_{1}+h_{1}, t_{c}-\varepsilon l_{1}+h_{2}\right]$, or until the final time $T$ is reached. The choice is made so that the interval is smallest. The interval $\left[t_{c}-\varepsilon l_{1}, T\right]$ is broken up in this manner so that, as explained before, we can treat the delay differential equation as an ordinary differential equation on each of these subintervals. When we reach any of the $t_{c}-\varepsilon l_{1}+h_{j}(j=2, \ldots, s)$ before proceeding a length $h_{1}$, then we derive new versions of equations (24) and (25). This is necessary because in each of the intervals $\left[t_{c}-\varepsilon l_{1}+h_{j}, t_{c}+\varepsilon l_{2}+h_{j}\right](j=1, \ldots, s)$, the time delays in the state equations have the effect of perturbing the system. These new equations, replacing (24) and (25), are

where

$$
x_{i}\left(u_{\varepsilon}^{1}\right)\left(t_{c}+\varepsilon l_{2}+h_{j}\right)=x_{i}\left(u^{1}\right)\left(t_{c}+\varepsilon l_{2}+h_{j}\right)+\varepsilon \xi_{i}\left(u^{1}\right)\left(t_{c}+\varepsilon l_{2}+h_{j}\right)+o(\varepsilon)
$$

$$
\begin{aligned}
\xi_{i}\left(u^{1}\right)\left(t_{c}+\varepsilon l_{2}+h_{j}\right)=\delta & x_{i}\left(u^{1}\right)\left(t_{c}-\varepsilon l_{1}+h_{j}\right) \\
& +\left(l_{1}+l_{2}\right)\left[f_{i}^{j}\left(t_{c}+\varepsilon l_{2}, x\left(u^{1}\right)\left(t_{c}+\varepsilon l_{2}\right), V_{u^{1}}\left(t_{c}+\varepsilon l_{2}\right)\right)\right. \\
& \left.-f_{i}^{j}\left(t_{c}+\varepsilon l_{2}, x\left(u^{1}\right)\left(t_{c}+\varepsilon l_{2}\right), u^{1}\left(t_{c}+\varepsilon l_{2}\right)\right)\right], \\
& \quad i=0,1, \ldots, n ; j=0,1, \ldots, s .
\end{aligned}
$$

Using this, and piecing together the $\delta x_{i}$ we arrive at the following formulae

$$
\begin{aligned}
x_{i}\left(u_{s}^{1}\right)(t) & =x_{i}\left(u^{1}\right)(t)+\varepsilon \delta x_{i}\left(u^{1}\right)(t)+o(\varepsilon), \\
\delta \dot{x}_{i}\left(u^{1}\right)(t) & =\sum_{k=0}^{n} \sum_{j=0}^{s} \frac{\partial f_{i}^{j}\left(t-h_{j}, x\left(u^{1}\right)\left(t-h_{j}\right), u^{1}\left(t-h_{j}\right)\right)}{\partial x_{k}} . \delta x_{k}\left(u^{1}\right)\left(t-h_{j}\right)
\end{aligned}
$$

for all $t \in[0, T]$ except $t \in\left(t_{c}-\varepsilon l_{1}+h_{j}, t_{c}+\varepsilon l_{2}+h_{j}\right], j=0,1, \ldots, s$, where (32) is updated by the "initial" conditions

$$
\begin{aligned}
& \delta x_{i}\left(u^{1}\right)\left(t+h_{f}\right)=\delta x_{i}\left(u^{1}\right)\left(t_{c}-\varepsilon l_{1}+h_{j}\right)+\left(l_{1}+l_{2}\right) \\
& \times\left[f_{i}^{j}\left(t_{c}+\varepsilon l_{2}, x\left(u^{1}\right)\left(t_{c}+\varepsilon l_{2}\right), V_{u^{1}}\left(t_{c}+\varepsilon l_{2}\right)\right)\right. \\
&\left.-f_{i}^{j}\left(t_{c}+\varepsilon l_{2}, x\left(u^{1}\right)\left(t_{c}+\varepsilon l_{2}\right), u^{1}\left(t_{c}+\varepsilon l_{2}\right)\right)\right], \\
& t \in\left(t_{c}-\varepsilon l_{1}, t_{c}+\varepsilon l_{2}\right],
\end{aligned}
$$


where $j=0,1, \ldots, s$ and

$$
\delta x_{i}\left(u^{1}\right)(t)=0 \quad \text { for } t \in\left[0, t_{c}-\varepsilon l_{1}\right], \quad i=0,1, \ldots, n .
$$

REMARK 4.2. The solution $\delta x_{i}(t)$ of the variational system (32)-(34) is continuous and differentiable everywhere on $[0, T]$ except possibly at $\theta \in \Theta\left(u^{1}\right)$ and on the intervals $\left(t_{c}-\varepsilon l_{1}+h_{j}, t_{c}+\varepsilon l_{2}+h_{j}\right], j=0,1, \ldots, s$.

Let $\psi\left(u^{1}\right)$ be the solution of the adjoint system (4)-(5) due to the control $u^{1}$ and define

$$
Z\left(u^{1}\right)(t)=\sum_{i=0}^{n} \psi_{i}\left(u^{1}\right)(t) . \delta x_{i}\left(u^{1}\right)(t)
$$

Then, it is clear that $Z\left(u^{1}\right)$ is continuous and also differentiable on $[0, T]$ except possibly on $\left(t_{c}-\varepsilon l_{1}+h_{j}, t_{c}+\varepsilon l_{2}+h_{j}\right], j=0,1, \ldots, s$, and at $\theta \in \Theta\left(u^{1}\right)$.

The following Lemma is an extension of assertion (2.21) found in [2].

LEMMA 4.3.

$$
\begin{gathered}
Z\left(u^{1}\right)(T)=Z\left(u^{1}\right)\left(t_{c}+\varepsilon l_{2}\right)+\left(l_{1}+l_{2}\right) \sum_{j=1}^{s} \sum_{i=0}^{n} \psi_{i}\left(u^{1}\right)\left(t_{c}+\varepsilon l_{2}+h_{j}\right) \\
\times\left[f_{i}^{j}\left(t_{c}+\varepsilon l_{2}, x\left(u^{1}\right)\left(t_{c}+\varepsilon l_{2}\right), V_{u^{1}}\left(t_{c}+\varepsilon l_{2}\right)\right)\right. \\
\left.\quad-f_{i}^{j}\left(t_{c}+\varepsilon l_{2}, x\left(u^{1}\right)\left(t_{c}+\varepsilon l_{2}\right), u^{1}\left(t_{c}+\varepsilon l_{2}\right)\right)\right]+o(1),
\end{gathered}
$$

where $o(1)$ is such that $\lim _{\epsilon \downarrow 0}\|o(1)\|=0$.

Proof. We have

$$
\begin{aligned}
& Z\left(u^{1}\right)(T)-Z\left(u^{1}\right)\left(t_{c}+\varepsilon l_{2}\right)= \\
& \quad \int_{l_{c}+\varepsilon l_{2}}^{t_{0}-\varepsilon l_{1}+h_{1}} \frac{d Z}{d t}\left(u^{1}\right)(t) d t+Z\left(u^{1}\right)\left(t_{c}+\varepsilon l_{2}+h_{1}\right)-Z\left(u^{1}\right)\left(t_{c}-\varepsilon l_{1}+h_{1}\right) \\
& \quad+\int_{l_{c}+\varepsilon l_{2}+h_{1}}^{t_{0}-\epsilon l_{1}+h_{2}} \frac{d Z}{d t}\left(u^{1}\right)(t) d t+\ldots+Z\left(u^{1}\right)\left(t_{c}+\varepsilon l_{2}+h_{s}\right)-Z\left(u^{1}\right)\left(t_{c}-\varepsilon l_{1}+h_{s}\right) \\
& \quad+\int_{t_{c}+\varepsilon l_{2}+h_{s}}^{T} \frac{d Z}{d t}\left(u^{1}\right)(t) d t
\end{aligned}
$$

and

$$
\frac{d Z}{d t}\left(u^{1}\right)(t)=\sum_{i=0}^{n} \psi_{i}\left(u^{1}\right)(t) . \delta x_{i}\left(u^{1}\right)(t)+\sum_{i=0}^{n} \psi_{i}\left(u^{1}\right)(t) . \delta \dot{x}_{i}\left(u^{1}\right)(t)
$$

exists everywhere in $\left[t_{c}+\varepsilon l_{2}, T\right]$ except possibly on $\left(t_{c}-\varepsilon l_{1}+h_{j}, t_{c}+\varepsilon l_{2}+h_{j}\right]$, $j=0,1, \ldots, s$, and at $\theta \in \Theta\left(u^{1}\right)$.

If we take $s=2$, no generality will be lost and the proof will be clearer for the lack of repetition. 
To simplify matters we now define

$$
\begin{aligned}
M_{i \alpha}^{j}(t) \triangleq \frac{\partial f_{i}^{j}\left(t, x\left(u^{1}\right)(t), u^{1}(t)\right)}{\partial x_{\alpha}} . \delta x_{\alpha}\left(u^{1}\right)(t), \\
j=0,1,2 ; \quad i=0,1, \ldots, n ; \quad \alpha=0,1, \ldots, n .
\end{aligned}
$$

Equation (37) now becomes

$$
\begin{aligned}
& Z\left(u^{1}\right)(T)-Z\left(u^{1}\right)\left(t_{c}+\varepsilon l_{2}\right) \\
& =\sum_{\alpha=0}^{n} \sum_{i=0}^{n} \int_{t_{c}+c h_{s}}^{t_{c}-t_{1}+h_{1}} \psi_{i}\left(u^{1}\right)(t)\left[M_{i \alpha}^{0}(t)+M_{i \alpha}^{1}\left(t-h_{1}\right)+M_{i \alpha}^{2}\left(t-h_{2}\right)\right] d t \\
& -\sum_{\alpha=0}^{n} \sum_{i=0}^{n} \int_{l_{0}+\varepsilon l_{2}}^{l_{c}-\varepsilon l_{1}+h_{1}}\left[\psi_{i}\left(u^{1}\right)(t) M_{i \alpha}^{0}(t)+\psi_{i}\left(u^{1}\right)\left(t+h_{1}\right) M_{i \alpha}^{1}(t)\right. \\
& \left.+\psi_{i}\left(u^{1}\right)\left(t+h_{2}\right) M_{i \alpha}^{2}(t)\right] d t \\
& +\sum_{\alpha=0}^{n} \sum_{i=0}^{n} \int_{l_{c}+\varepsilon l_{2}+h_{1}}^{t_{c}-\epsilon l_{1}+h_{\alpha}} \psi_{i}\left(u^{1}\right)(t)\left[M_{i \alpha}^{0}(t)+M_{i \alpha}^{1}\left(t-h_{1}\right)+M_{i \alpha}^{2}\left(t-h_{2}\right)\right] d t \\
& -\sum_{\alpha=0}^{n} \sum_{i=0}^{n} \int_{b_{0}+e_{2}+h_{1}}^{t_{e}-\varepsilon l_{1}+h_{2}}\left[\psi_{i}\left(u^{1}\right)(t) M_{i \alpha}^{0}(t)+\psi_{i}\left(u^{1}\right)\left(t+h_{1}\right) M_{i \alpha}^{1}(t)\right. \\
& \left.+\psi_{i}\left(u^{1}\right)\left(t+h_{2}\right) M_{i \alpha}^{2}(t)\right] d t \\
& +\sum_{\alpha=0}^{n} \sum_{i=0}^{n} \int_{t_{c}+\epsilon_{2}+h_{3}}^{T} \psi_{i}\left(u^{1}\right)(t)\left[M_{i \alpha}^{0}(t)+M_{i \alpha}^{1}\left(t-h_{1}\right)+M_{i \alpha}^{2}\left(t-h_{2}\right)\right] d t \\
& -\sum_{\alpha=0}^{n} \sum_{i=0}^{n} \int_{t_{0}+c_{2}+h_{3}}^{T}\left[\psi_{i}\left(u^{1}\right)(t) M_{i \alpha}^{0}(t)+\psi_{i}\left(u^{1}\right)\left(t+h_{1}\right) M_{i \alpha}^{1}(t)\right. \\
& \left.+\psi_{i}\left(u^{1}\right)\left(t+h_{2}\right) M_{i \alpha}^{2}(t)\right] d t \\
& +Z\left(u^{1}\right)\left(t_{c}+\varepsilon l_{2}+h_{1}\right)-Z\left(u^{1}\right)\left(t_{c}-\varepsilon l_{1}+h_{1}\right) \\
& +Z\left(u^{1}\right)\left(t_{c}+\varepsilon l_{2}+h_{2}\right)-Z\left(u^{1}\right)\left(t_{c}-\varepsilon l_{1}+h_{2}\right) \text {. }
\end{aligned}
$$

Since $\delta x_{i}\left(u^{1}\right)(t)=0$ for $t \in\left[0, t_{c}+\varepsilon l_{2}\right)$ and $\psi_{i}\left(u^{1}\right)(t)=0$ for $t>T$, it follows, after changing the variable in some of the integrals to $t^{\prime}=t-h_{j}, j=1,2$, and then dropping the prime, that

$$
\begin{aligned}
Z\left(u^{1}\right)(T) & -Z\left(u^{1}\right)\left(t_{c}+\varepsilon l_{2}\right)-Z\left(u^{1}\right)\left(t_{c}+\varepsilon l_{2}+h_{1}\right)+Z\left(u^{1}\right)\left(t_{c}-\varepsilon l_{1}+h_{1}\right) \\
& -Z\left(u^{1}\right)\left(t_{c}+\varepsilon l_{2}+h_{2}\right)+Z\left(u^{1}\right)\left(t_{c}-\varepsilon l_{1}+h_{2}\right) \\
= & -\sum_{\alpha=0}^{n} \sum_{i=0}^{n} \int_{t_{c}+\varepsilon l_{2}}^{t_{c}-\varepsilon l_{1}+h_{2}}\left[\psi_{i}\left(u^{1}\right)\left(t+h_{1}\right) M_{i \alpha}^{1}(t)+\psi_{i}\left(u^{1}\right)\left(t+h_{2}\right) M_{i \alpha}^{2}(t)\right] d t \\
+ & \sum_{\alpha=0}^{n} \sum_{i=0}^{n} \int_{t_{c}+\varepsilon l_{2}}^{t_{c}-\varepsilon l_{1}+h_{2}-h_{1}} \psi_{i}\left(u^{1}\right)\left(t+h_{1}\right) M_{i \alpha}^{1}(t) d t
\end{aligned}
$$




$$
\begin{aligned}
& -\sum_{\alpha=0}^{n} \sum_{i=0}^{n} \int_{t_{c}+\varepsilon l_{2}+h_{1}}^{t_{c}-\varepsilon l_{1}+h_{2}}\left[\psi_{i}\left(u^{1}\right)\left(t+h_{1}\right) M_{i \alpha}^{1}(t)+\psi_{i}\left(u^{1}\right)\left(t+h_{2}\right) M_{i \alpha}^{2}(t)\right] d t \\
& +\sum_{\alpha=0}^{n} \sum_{i=0}^{n} \int_{t_{c}+l_{2}+h_{2}-h_{1}}^{T-h_{1}} \psi_{i}\left(u^{1}\right)\left(t+h_{1}\right) M_{i \alpha}^{1}(t) d t \\
& +\sum_{\alpha=0}^{n} \sum_{i=0}^{n} \int_{l_{c}+\varepsilon l_{2}}^{T-h_{2}} \psi_{i}\left(u^{1}\right)\left(t+h_{2}\right) M_{i \alpha}^{2}(t) d t \\
& -\sum_{\alpha=0}^{n} \sum_{i=0}^{n} \int_{i_{c}+c h_{2}+h_{2}}^{T-h_{1}} \psi_{i}\left(u^{1}\right)\left(t+h_{1}\right) M_{i \alpha}^{1}(t) d t \\
& -\sum_{\alpha=0}^{n} \sum_{i=0}^{n} \int_{l_{c}+\epsilon l_{2}+h_{1}}^{T-h_{2}} \psi_{i}\left(u^{1}\right)\left(t+h_{2}\right) M_{i \alpha}^{2}(t) d t \\
& =-\sum_{\alpha=0}^{n} \sum_{i=0}^{n}\left[\int_{t_{c}+\epsilon l_{2}}^{t_{c}-\varepsilon l_{1}+h_{1}}+\int_{t_{c}+\epsilon l_{2}+h_{1}}^{t_{c}-\epsilon l_{1}+h_{2}}+\int_{t_{c}+\epsilon l_{2}+h_{2}}^{T-h_{1}}\right] \psi_{i}\left(u^{1}\right)\left(t+h_{1}\right) M_{i \alpha}^{1}(t) d t \\
& +\sum_{\alpha=0}^{n} \sum_{i=0}^{n}\left[\int_{t_{a}+\epsilon l_{2}}^{t_{c}-\epsilon l_{1}+h_{2}-h_{1}}+\int_{t_{c}+\epsilon l_{2}+h_{2}-h_{1}}^{T-h_{1}}\right] \psi_{i}\left(u^{1}\right)\left(t+h_{1}\right) M_{i \alpha}^{1}(t) d t \\
& -\sum_{\alpha=0}^{n} \sum_{i=0}^{n}\left[\int_{t_{c}+\varepsilon l_{2}}^{t_{c}-\epsilon l_{1}+h_{1}}+\int_{l_{c}+\varepsilon l_{2}+h_{1}}^{t_{c}-\varepsilon l_{1}+h_{2}}+\int_{l_{a}+\varepsilon l_{2}+h_{2}}^{T-h_{2}}\right] \psi_{i}\left(u^{1}\right)\left(t+h_{2}\right) M_{i \alpha}^{2}(t) d t \\
& +\sum_{\alpha=0}^{n} \sum_{i=0}^{n} \int_{t_{c}+l_{2}}^{T-h_{2}} \psi_{i}\left(u^{1}\right)\left(t+h_{2}\right) M_{i \alpha}^{2}(t) d t \text {. }
\end{aligned}
$$

Now these terms cancel each other except for various intervals of length $\varepsilon\left(l_{1}+l_{2}\right)$. But since $\psi\left(u^{1}\right)(t)$ is an absolutely continuous function and the partial derivatives

$$
\frac{\partial f_{i}^{j}\left(t, x\left(u^{1}\right)(t), u^{1}(t)\right)}{\partial x_{\alpha}}, \quad i=0,1, \ldots, n ; j=0,1,2
$$

along with the $\delta x_{\alpha}\left(u^{1}\right)(t), \alpha=0,1, \ldots, n$, are piecewise continuous, it follows that the integrals cancel to within $o(1)$. This leaves us with

$$
\begin{aligned}
Z\left(u^{1}\right)(T)-Z\left(u^{1}\right)\left(t_{c}+\varepsilon l_{2}\right)= & Z\left(u^{1}\right)\left(t_{c}+\varepsilon l_{2}+h_{1}\right)-Z\left(u^{1}\right)\left(t_{c}-\varepsilon l_{1}+h_{1}\right) \\
& +Z\left(u^{1}\right)\left(t_{c}+\varepsilon l_{2}+h_{2}\right)-Z\left(u^{1}\right)\left(t_{c}-\varepsilon l_{1}+h_{2}\right)+o(1) .
\end{aligned}
$$

Using the definition of $\mathcal{Z}\left(u^{1}\right)(t)$ and equation (33), we obtain

$$
\begin{gathered}
Z\left(u^{1}\right)(T)-Z\left(u^{1}\right)\left(t_{c}+\varepsilon l_{2}\right)=\sum_{i=0}^{n} \psi_{i}\left(u^{1}\right)\left(t_{c}+\varepsilon l_{2}+h_{1}\right) \\
\times\left[\delta x_{i}\left(u^{1}\right)\left(t_{c}-\varepsilon l_{1}+h_{1}\right)+\left(l_{1}+l_{2}\right)\left\{f_{i}^{1}\left(t_{c}+\varepsilon l_{2}, x\left(u^{1}\right)\left(t_{c}+\varepsilon l_{2}\right), V_{u^{1}}\left(t_{c}+\varepsilon l_{2}\right)\right)\right.\right. \\
\left.\left.-f_{i}^{1}\left(t_{c}+\varepsilon l_{2}, x\left(u^{1}\right)\left(t_{c}+\varepsilon l_{2}\right), u^{1}\left(t_{c}+\varepsilon l_{2}\right)\right)\right\}\right]
\end{gathered}
$$




$$
\begin{aligned}
& -\sum_{i=0}^{n} \psi_{i}\left(u^{1}\right)\left(t_{c}-\varepsilon l_{1}+h_{1}\right) \cdot \delta x_{i}\left(u^{1}\right)\left(t_{c}-\varepsilon l_{1}+h_{1}\right)+\sum_{i=0}^{n} \psi_{i}\left(u^{1}\right)\left(t_{c}+\varepsilon l_{2}+h_{2}\right) \\
& \times\left[\delta x_{i}\left(u^{1}\right)\left(t_{c}-\varepsilon l_{1}+h_{2}\right)+\left(l_{1}+l_{2}\right)\left\{f_{i}^{2}\left(t_{c}+\varepsilon l_{2}, x\left(u^{1}\right)\left(t_{c}+\varepsilon l_{2}\right), V_{u^{1}}\left(t_{c}+\varepsilon l_{2}\right)\right)\right.\right. \\
& \left.\left.\quad-f_{i}^{2}\left(t_{c}+\varepsilon l_{2}, x\left(u^{1}\right)\left(t_{c}+\varepsilon l_{2}\right), u^{1}\left(t_{c}+\varepsilon l_{2}\right)\right)\right\}\right] \\
& -\sum_{i=0}^{n} \psi_{i}\left(u^{1}\right)\left(t_{c}-\varepsilon l_{1}+h_{2}\right) \cdot \delta x_{i}\left(u^{1}\right)\left(t_{c}-\varepsilon l_{1}+h_{2}\right)+o(1) .
\end{aligned}
$$

But, as was noted before, $\psi\left(u^{1}\right)(t)$ is an absolutely continuous function, so we can replace $\psi\left(u^{1}\right)\left(t_{c}-\varepsilon l_{1}+h_{j}\right)$ by $\psi\left(u^{1}\right)\left(t_{c}+\varepsilon l_{2}+h_{j}\right)$ and the difference will be absorbed in the $o(1)$ terms. Therefore

$$
\begin{aligned}
Z\left(u^{1}\right)(T)=Z\left(u^{1}\right)\left(t_{c}+\right. & \left.\varepsilon l_{2}\right)+\left(l_{1}+l_{2}\right) \sum_{j=1}^{2} \sum_{i=0}^{n} \psi_{i}\left(u^{1}\right)\left(t_{c}+\varepsilon l_{2}+h_{j}\right) \\
& \times\left[f_{i}^{j}\left(t_{c}+\varepsilon l_{2}, x\left(u^{1}\right)\left(t_{c}+\varepsilon l_{2}\right), V_{u^{1}}\left(t_{c}+\varepsilon l_{2}\right)\right)\right. \\
& \left.-f_{i}^{j}\left(t_{c}+\varepsilon l_{2}, x\left(u^{1}\right)\left(t_{c}+\varepsilon l_{2}\right), u^{1}\left(t_{c}+\varepsilon l_{2}\right)\right)\right]+o(1)
\end{aligned}
$$

which is (36) with $s=2$. The Lemma is now proved.

THEOREM 4.4. Consider the problem $P$. Suppose that the assumptions $(A)$ and $\left(A_{H}\right)$ are satisfied and that $u^{1} \in D$ is not an extremal control. Let $t_{c} \in[0, T]$ be such that $V_{u^{1}}\left(t_{c}\right) \neq u^{1}\left(t_{c}\right)$, let

$$
\Omega_{\epsilon}=\left[t_{c}-\varepsilon l_{1}, t_{c}+\varepsilon l_{2}\right]
$$

and

$$
u_{\epsilon}^{1}(t)= \begin{cases}V_{u^{1}}(t) & \text { if } t \in \Omega_{\epsilon}, \\ u^{1}(t) & \text { elsewhere, }\end{cases}
$$

where $l_{1}=0$ if $t_{c}=0$ or if $t_{c} \in \Theta\left(u^{1}\right)$ and $l_{2}>0$, and $l_{1}$ is a positive constant elsewhere; and $l_{2}=0$ if $t_{c}=T$ or if $t_{c} \in \Theta\left(u^{1}\right)$ and $l_{1}>0$, and $l_{2}$ is a positive constant elsewhere. $\Theta\left(u^{1}\right)$ is as defined in Remark 3.6.

Then

$$
\begin{aligned}
J\left(u^{1}\right)-J\left(u_{e}^{1}\right)=\int_{\Omega_{e}}\left\{H_{\max }\left(t, x\left(u^{1}\right)(t), \Psi\left(u^{1}\right)(t)\right)\right. \\
\left.\quad-H\left(t, x\left(u^{1}\right)(t), u^{1}(t), \Psi\left(u^{1}\right)(t)\right)\right\} d t+o(\varepsilon),
\end{aligned}
$$

where $H_{\max }$ is as defined in Remark 3.7 and $o(\varepsilon)$ is such that $\lim _{\varepsilon \downarrow 0}\|o(\varepsilon)\| / \varepsilon=0$. Further, there exists an $\varepsilon_{0}>0$ such that

$$
J\left(u^{1}\right)>J\left(u_{\varepsilon}^{1}\right)
$$

whenever $0<\varepsilon<\varepsilon_{0}$. 
Proof. We have

$$
\begin{aligned}
& J\left(u^{1}\right)=x_{0}\left(u^{1}\right)(T), \\
& J\left(u_{\varepsilon}^{1}\right)=x_{0}\left(u_{\varepsilon}^{1}\right)(T) .
\end{aligned}
$$

Thus, it follows from equation (31) that

$$
\begin{aligned}
J\left(u^{1}\right)-J\left(u_{\varepsilon}^{1}\right) & =x_{0}\left(u^{1}\right)(T)-x_{0}\left(u_{\varepsilon}^{1}\right)(T) \\
& =-\varepsilon \delta x_{0}(T)+o(\varepsilon) .
\end{aligned}
$$

However, $\psi_{i}\left(u^{1}\right)(T)=0, i=1, \ldots, n$. Therefore,

$$
\begin{aligned}
J\left(u^{1}\right)-J\left(u_{\varepsilon}^{1}\right) & =\varepsilon\left[-\delta x_{0}\left(u^{1}\right)(T)+\sum_{i=1}^{n} \delta x_{i}\left(u^{1}\right)(T) \cdot \psi_{i}\left(u^{1}\right)(T)\right]+o(\varepsilon) \\
& =\varepsilon Z\left(u^{1}\right)(T)+o(\varepsilon) .
\end{aligned}
$$

By virtue of relation (36), (45) reduces to

$$
\begin{aligned}
& J\left(u^{1}\right)-J\left(u_{\varepsilon}^{1}\right)=\varepsilon \mathcal{(}\left(u^{1}\right)\left(t_{c}+\varepsilon l_{2}\right) \\
&+ \varepsilon\left(l_{1}+l_{2}\right) \sum_{j=1}^{s} \sum_{i=0}^{n} \psi_{i}\left(u^{1}\right)\left(t_{c}+\varepsilon l_{2}+h_{j}\right) \\
& \times\left[f_{i}^{j}\left(t_{c}+\varepsilon l_{2}, x\left(u^{1}\right)\left(t_{c}+\varepsilon l_{2}\right), V_{u^{1}}\left(t_{c}+\varepsilon l_{2}\right)\right)\right. \\
&\left.-f_{i}^{j}\left(t_{c}+\varepsilon l_{2}, x\left(u^{1}\right)\left(t_{c}+\varepsilon l_{2}\right), u^{1}\left(t_{c}+\varepsilon l_{2}\right)\right)\right]+o(\varepsilon) \\
&=\varepsilon\left(l_{1}+l_{2}\right) \sum_{j=0}^{s} \sum_{i=0}^{n} \psi_{i}\left(t_{c}+\varepsilon l_{2}+h_{j}\right) \\
& \quad \times\left[f_{i}^{j}\left(t_{c}+\varepsilon l_{2}, x\left(u^{1}\right)\left(t_{c}+\varepsilon l_{2}\right), V_{u^{1}}\left(t_{c}+\varepsilon l_{2}\right)\right)\right. \\
&\left.-f_{i}^{j}\left(t_{c}+\varepsilon l_{2}, x\left(u^{1}\right)\left(t_{c}+\varepsilon l_{2}\right), u^{1}\left(t_{c}+\varepsilon l_{2}\right)\right)\right]+o(\varepsilon) \\
&=\varepsilon\left(l_{1}+l_{2}\right)\left\{H\left(t_{c}+\varepsilon l_{2}, x\left(u^{1}\right)\left(t_{c}+\varepsilon l_{2}\right), V_{u^{1}}\left(t_{c}+\varepsilon l_{2}\right), \Psi\left(u^{1}\right)\left(t_{c}+\varepsilon l_{2}\right)\right)\right. \\
&\left.-H\left(t_{c}+\varepsilon l_{2}, x\left(u^{1}\right)\left(t_{c}+\varepsilon l_{2}\right), u^{1}\left(t_{c}+\varepsilon l_{2}\right), \Psi\left(u^{1}\right)\left(t_{c}+\varepsilon l_{2}\right)\right)\right\}+o(\varepsilon) \\
&=\varepsilon\left(l_{1}+l_{2}\right)\left\{\left[H\left(t_{c}+\varepsilon l_{2}, x\left(u^{1}\right)\left(t_{c}+\varepsilon l_{2}\right), V_{u^{1}}\left(t_{c}+\varepsilon l_{2}\right), \Psi\left(u^{1}\right)\left(t_{c}+\varepsilon l_{2}\right)\right)\right.\right. \\
&\left.-H\left(t, x\left(u^{1}\right)(t), V_{u^{1}}(t), \Psi\left(u^{1}\right)(t)\right)\right] \\
&+\left[H\left(t, x\left(u^{1}\right)(t), V_{u^{1}}(t), \Psi\left(u^{1}\right)(t)\right)-H\left(t, x\left(u^{1}\right)(t), u^{1}(t), \Psi\left(u^{1}\right)(t)\right)\right] \\
&+\left[H\left(t, x\left(u^{1}\right)(t), u^{1}(t), \Psi\left(u^{1}\right)(t)\right)\right. \\
&\left.\left.-H\left(t_{c}+\varepsilon l_{2}, x\left(u^{1}\right)\left(t_{c}+\varepsilon l_{2}\right), u^{1}\left(t_{c}+\varepsilon l_{2}\right), \Psi\left(u^{1}\right)\left(t_{c}+\varepsilon l_{2}\right)\right)\right]\right\}+o(\varepsilon)
\end{aligned}
$$




$$
\begin{aligned}
=\int_{t_{c}-\varepsilon l_{1}}^{t_{\varepsilon}+\varepsilon l_{2}}\left[H\left(t_{c}+\varepsilon l_{2}, x\left(u^{1}\right)\left(i_{c}+\varepsilon l_{2}\right), V_{u^{1}}\left(t_{c}+\varepsilon l_{2}\right), \Psi\left(u^{1}\right)\left(t_{c}+\varepsilon l_{2}\right)\right)\right. \\
\left.\quad-H\left(t, x\left(u^{1}\right)(t), V_{u^{1}}(t), \Psi\left(u^{1}\right)(t)\right)\right] d t \\
\quad+\int_{t_{c}-\varepsilon l_{1}}^{t_{c}+\varepsilon l_{2}}\left[H\left(t, x\left(u^{1}\right)(t), V_{u^{1}}(t), \Psi\left(u^{1}\right)(t)\right)\right. \\
\left.\quad-H\left(t, x\left(u^{1}\right)(t), u^{1}(t), \Psi\left(u^{1}\right)(t)\right)\right] d t \\
\quad+\int_{t_{c}-\varepsilon l_{1}}^{t_{c}+\varepsilon l_{2}}\left[H\left(t, x\left(u^{1}\right)(t), u^{1}(t), \Psi\left(u^{1}\right)(t)\right)\right. \\
\left.\quad-H\left(t_{c}+\varepsilon l_{2}, x\left(u^{1}\right)\left(t_{c}+\varepsilon l_{2}\right), u^{1}\left(t_{c}+\varepsilon l_{2}\right), \Psi\left(u^{1}\right)\left(t_{c}+\varepsilon l_{2}\right)\right)\right] d t+o(\varepsilon) .
\end{aligned}
$$

Since

$$
H\left(\cdot, x\left(u^{1}\right)(\cdot), u^{1}(\cdot), \Psi\left(u^{1}\right)(\cdot)\right) \triangleq H^{1}(\cdot)
$$

and

$$
H\left(\cdot, x\left(u^{1}\right)(\cdot), V_{u^{1}}(\cdot), \Psi\left(u^{1}\right)(\cdot)\right) \triangleq H^{2}(\cdot)
$$

are continuous on $\left(t_{c}-\varepsilon l_{1}, t_{c}+\varepsilon l_{2}\right)$, it follows from the mean value theorem for integrals that

$$
\begin{aligned}
\int_{l_{c}-\varepsilon l_{1}}^{t_{c}+\varepsilon l_{2}}\left[H^{1}(t)-H^{1}\left(t_{c}+\varepsilon l_{2}\right)\right] d t-\int_{l_{c}-\varepsilon l_{1}}^{t_{c}+\varepsilon l_{2}}\left[H^{2}(t)-H^{2}\left(t_{c}+\varepsilon l_{2}\right)\right] d t \\
=\varepsilon\left(l_{1}+l_{2}\right)\left\{\left(H^{1}\left(\tau_{1}\right)-H^{1}\left(t_{c}+\varepsilon l_{2}\right)\right)-\left(H^{2}\left(\tau_{2}\right)-H^{2}\left(t_{c}+\varepsilon l_{2}\right)\right)\right\},
\end{aligned}
$$

where $\tau_{i} \in\left(t_{c}-\varepsilon l_{1}, t_{c}+\varepsilon l_{2}\right), i=1,2$.

Since $\varepsilon \rightarrow 0$ implies $\tau_{i} \rightarrow t_{c}$ and $t_{c}+\varepsilon l_{2} \rightarrow t_{c}$ it follows that $\left(H^{1}\left(\tau_{1}\right)-H^{1}\left(t_{c}+\varepsilon l_{2}\right)\right) \rightarrow 0$ and $\left(H^{2}\left(\tau_{2}\right)-H^{2}\left(t_{c}+\varepsilon l_{2}\right)\right) \rightarrow 0$ as $\varepsilon \rightarrow 0$. Thus

$$
\begin{aligned}
J\left(u^{1}\right)-J\left(u_{\ell}^{1}\right)=\int_{t_{c}-\varepsilon l_{l}}^{t_{c}+e l_{2}}\left[H\left(t, x\left(u^{1}\right)(t), V_{u^{1}}(t), \Psi\left(u^{1}\right)(t)\right)\right. \\
\\
\left.\quad-H\left(t, x\left(u^{1}\right)(t), u^{1}(t), \Psi\left(u^{1}\right)(t)\right)\right] d t+o(\varepsilon) .
\end{aligned}
$$

This proves the first part of the theorem.

Now it remains to show that the second statement is true. For this, we note that

$$
H\left(t_{c}, x\left(u^{1}\right)\left(t_{c}\right), V_{u^{1}}\left(t_{c}\right), \Psi\left(u^{1}\right)\left(t_{c}\right)\right)>H\left(t_{c}, x\left(u^{1}\right)\left(t_{c}\right), u^{1}\left(t_{c}\right), \Psi\left(u^{1}\right)\left(t_{c}\right)\right)
$$

and that the functions

$$
H\left(\cdot, x\left(u^{1}\right)(\cdot), u^{1}(\cdot), \Psi\left(u^{1}\right)(\cdot)\right) \text { and } H\left(\cdot, x\left(u^{1}\right)(\cdot), V_{u^{1}}(\cdot), \Psi\left(u^{1}\right)(\cdot)\right)
$$

are continuous on $\left(t_{c}-\varepsilon l_{1}, t_{c}+\varepsilon l_{2}\right)$. 
Thus, by an appropriate choice of $\varepsilon_{0}>0$, relation (47) implies that

$$
\frac{1}{\varepsilon\left(l_{1}+l_{2}\right)}\left[J\left(u^{1}\right)-J\left(u_{\varepsilon}^{1}\right)\right]>0
$$

for all $0<\varepsilon<\varepsilon_{0}$.

This completes the proof.

With the help of Theorem 4.4, the well-known necessary conditions for optimality given in Theorem 3.4, can be easily derived.

Proof of Theorem 3.4. Suppose $u^{1}$ is non-extremal. Then by the first statement of Theorem 4.4, we can find a $u_{\varepsilon}^{1}$, as defined in (41), such that

$$
\begin{aligned}
& J\left(u^{1}\right)-J\left(u_{s}^{1}\right)=\int_{\Omega_{\varepsilon}}\left\{H_{\max }\left(t, x\left(u^{1}\right)(t), \Psi\left(u^{1}\right)(t)\right)\right. \\
&\left.-H\left(t, x\left(u^{1}\right)(t), u^{1}(t), \Psi\left(u^{1}\right)(t)\right)\right\} d t+o(\varepsilon) .
\end{aligned}
$$

If we now suppose that, given an optimal control $u^{*}$, we can find a control $V_{u^{*}}$, which maximizes $H\left(t, x\left(u^{1}\right)(t), v, \Psi\left(u^{1}\right)(t)\right)$ on an $\Omega_{\varepsilon}$ but which, of course, cannot improve the cost functional $J$, then equation (49) will still hold and this will give

$$
\begin{aligned}
0>J\left(u^{*}\right)-J\left(u_{s}^{*}\right)=\int_{\Omega_{s}}\left\{H\left(t, x\left(u^{*}\right)(t), V_{u^{*}}(t), \Psi\left(u^{*}\right)(t)\right)\right. \\
\left.\quad-H\left(t, x\left(u^{*}\right)(t), u^{*}(t), \Psi\left(u^{*}\right)(t)\right)\right\} d t+o(\varepsilon) .
\end{aligned}
$$

By precisely the same argument as that used to obtain (48), we have

$$
\int_{\Omega_{\varepsilon}} H\left(t, x\left(u^{*}\right)(t), u^{*}(t), \Psi\left(u^{*}\right)(t)\right) d t<\int_{\Omega_{\varepsilon}} H\left(t, x\left(u^{*}\right)(t), V_{u^{*}}(t), \Psi\left(u^{*}\right)(t)\right) d t
$$

for $\varepsilon>0$ sufficiently small. This is a contradiction and hence the proof is complete.

The main objection to Theorem 4.4 in using it in a computational algorithm is that it requires exact maximization of the Hamiltonian at an infinite number of points. For this reason, the following theorem obtains similar results to Theorem 4.4 , but needs only approximate maximization of the Hamiltonian at a single point. It incorporates nominally a sub-procedure $S$, iterated on an integer $\rho$, that obtains an approximate maximizing control. This control, $w_{\rho} \in D$, is chosen such that

$$
\left|H_{\max }(t, x(u)(t), \Psi(u)(t))-H\left(t, x(u)(t), w_{\rho}(u)(t), \Psi(u)(t)\right)\right| \leqslant k \Gamma^{\rho}<\varepsilon
$$

for a given $u \in D$ and fixed $k$ and $\Gamma$ with $\Gamma<1$. 
THEOREM 4.5. Consider the problem $P$ and suppose that the assumptions $(A)$ and $\left(A_{H}\right)$ are satisfied and that $u^{\mathrm{1}} \in D$ is not an extremal control. Let $t_{c} \in[0, T]$ be such that $V_{u^{1}}\left(t_{c}\right) \neq u^{1}\left(t_{c}\right)$. Define

and

$$
\Omega_{\varepsilon}=\left[t_{c}-\varepsilon l_{1}, t_{c}+\varepsilon l_{2}\right]
$$

$$
u_{s}^{1}(t)= \begin{cases}w_{\rho}\left(u^{1}\right)\left(t_{c}\right) & \text { if } t \in \Omega_{\varepsilon}, \\ u^{1}(t) & \text { elsewhere, }\end{cases}
$$

where $l_{1}=0$ if $t_{c}=0$ or if $t_{c} \in \Theta\left(u^{1}\right)$ and $l_{2}>0$, and $l_{1}$ is a positive constant elsewhere; and $l_{2}=0$ if $t_{c}=T$ or if $t_{c} \in \Theta\left(u^{1}\right)$ and $l_{1}>0$, and $l_{2}$ is a positive constant elsewhere. Further let $w_{\rho}\left(u^{1}\right)\left(t_{c}\right)$ be calculated in the sub-procedure $S$, previously mentioned, and satisfy relation (50) and let $\Theta\left(u^{1}\right)$ be as defined in Remark 3.6. Then,

$$
\begin{aligned}
J\left(u^{1}\right)-J\left(u_{s}^{1}\right)=\int_{\Omega_{s}}\left\{H \left(t, x\left(u^{1}\right)(t),\right.\right. & \left.w_{\rho}\left(u^{1}\right)\left(t_{c}\right), \Psi\left(u^{1}\right)(t)\right) \\
& \left.-H\left(t, x\left(u^{1}\right)(t), u^{1}(t), \Psi\left(u^{1}\right)(t)\right)\right\} d t+o(\varepsilon) .
\end{aligned}
$$

Further, there exists an $\varepsilon_{0}>0$ such that

$$
J\left(u^{1}\right)>J\left(u_{s}^{1}\right)
$$

for all integers $\rho$ such that $k \Gamma^{\rho}<\varepsilon$ where $\varepsilon$ is such that $0<\varepsilon<\varepsilon_{0}$.

Proof. In view of the hypotheses of Theorems 4.4 and 4.5, we note that their only difference is in the definition of $u_{\varepsilon}^{1}$. More precisely, in the hypotheses of this theorem, $u_{\varepsilon}^{1}(t)=w_{\rho}\left(u^{1}\right)\left(t_{c}\right)$ on $\Omega_{\varepsilon}$ instead of $u_{\varepsilon}^{1}(t)=V_{u^{1}}(t)$ on $\Omega_{\varepsilon}$ as defined in the hypotheses of Theorem 4.4. Thus, by using an argument similar to that for obtaining relation (46), we get

$$
\begin{gathered}
J\left(u^{1}\right)-J\left(u_{\varepsilon}^{1}\right)=\varepsilon\left(l_{1}+l_{2}\right)\left\{\left[H\left(t_{c}+\varepsilon l_{2}, x\left(u^{1}\right)\left(t_{c}+\varepsilon l_{2}\right), w_{\rho}\left(u^{1}\right)\left(t_{c}\right), \Psi\left(u^{1}\right)\left(t_{c}+\varepsilon l_{2}\right)\right)\right.\right. \\
\left.-H\left(t, x\left(u^{1}\right)(t), w_{\rho}\left(u^{1}\right)\left(t_{c}\right), \Psi\left(u^{1}\right)(t)\right)\right] \\
+\left[H\left(t, x\left(u^{1}\right)(t), w_{\rho}\left(u^{1}\right)\left(t_{c}\right), \Psi\left(u^{1}\right)(t)\right)\right. \\
\left.\quad-H\left(t, x\left(u^{1}\right)(t), u^{1}(t), \Psi\left(u^{1}\right)(t)\right)\right] \\
+\left[H\left(t, x\left(u^{1}\right)(t), u^{1}(t), \Psi\left(u^{1}\right)(t)\right)\right. \\
\left.\left.+o(\varepsilon) \quad-H\left(t_{c}+\varepsilon l_{2}, x\left(u^{1}\right)\left(t_{c}+\varepsilon l_{2}\right), u^{1}\left(t_{c}+\varepsilon l_{2}\right), \Psi\left(u^{1}\right)\left(t_{c}+\varepsilon l_{2}\right)\right)\right]\right\}
\end{gathered}
$$




$$
\begin{aligned}
& =\int_{t_{c}-\varepsilon l_{1}}^{t_{c}+\varepsilon l_{2}}\left[H\left(t_{c}+\varepsilon l_{2}, x\left(u^{1}\right)\left(t_{c}+\varepsilon l_{2}\right), w_{\rho}\left(u^{1}\right)\left(t_{c}\right), \Psi\left(u^{1}\right)\left(t_{c}+\varepsilon l_{2}\right)\right)\right. \\
& \left.\quad-H\left(t, x\left(u^{1}\right)(t), w_{\rho}\left(u^{1}\right)\left(t_{c}\right), \Psi\left(u^{1}\right)(t)\right)\right] d t \\
& +\int_{t_{c}-\varepsilon l_{1}}^{t_{c}+\varepsilon l_{2}}\left[H\left(t, x\left(u^{1}\right)(t), w_{\rho}\left(u^{1}\right)\left(t_{c}\right), \Psi\left(u^{1}\right)(t)\right)\right. \\
& \left.\quad-H\left(t, x\left(u^{1}\right)(t), u^{1}(t), \Psi\left(u^{1}\right)(t)\right)\right] d t \\
& +\int_{l_{c}-\varepsilon l_{1}}^{t_{c}+\varepsilon l_{2}}\left[H\left(t, x\left(u^{1}\right)(t), u^{1}(t), \Psi\left(u^{1}\right)(t)\right)\right. \\
& +o(\varepsilon) .
\end{aligned}
$$

Since

$$
H\left(\cdot, x\left(u^{1}\right)(\cdot), u^{1}(\cdot), \Psi\left(u^{1}\right)(\cdot)\right) \triangleq H^{1}(\cdot)
$$

and

$$
H\left(\cdot, x\left(u^{1}\right)(\cdot), w_{\rho}\left(u^{1}\right)\left(t_{c}\right), \Psi\left(u^{1}\right)(\cdot)\right) \triangleq H^{2}(\cdot)
$$

are continuous on $\left(t_{c}-\varepsilon l_{1}, t_{c}+\varepsilon l_{2}\right)$ it follows from the mean value theorem for integrals that

$$
\begin{array}{r}
\int_{t_{c}-\varepsilon l_{1}}^{t_{c}+\varepsilon l_{2}}\left[H^{1}(t)-H^{1}\left(t_{c}+\varepsilon l_{2}\right)\right] d t-\int_{l_{c}-\varepsilon l_{1}}^{t_{c}+\varepsilon l_{2}}\left[H^{2}(t)-H^{2}\left(t_{c}+\varepsilon l_{2}\right)\right] d t \\
=\varepsilon\left(l_{1}+l_{2}\right)\left\{\left(H^{1}\left(\tau_{1}\right)-H^{1}\left(t_{c}+\varepsilon l_{2}\right)\right)-\left(H^{2}\left(\tau_{2}\right)-H^{2}\left(t_{c}+\varepsilon l_{2}\right)\right)\right\},
\end{array}
$$

where $\tau_{i} \in\left(t_{c}-\varepsilon l_{1}, t_{c}+\varepsilon l_{2}\right), i=1,2$.

Since $\varepsilon \rightarrow 0$ implies $\tau_{i} \rightarrow t_{c}$ and $t_{c}+\varepsilon l_{2} \rightarrow t_{c}$, it follows that $\left(H^{1}\left(\tau_{1}\right)-H^{1}\left(t_{c}+\varepsilon l_{2}\right)\right) \rightarrow 0$ and $\left(H^{2}\left(\tau_{2}\right)-H^{2}\left(t_{c}+\varepsilon l_{2}\right)\right) \rightarrow 0$.

Thus,

$$
\begin{aligned}
J\left(u^{1}\right)-J\left(u_{\varepsilon}^{1}\right)=\int_{t_{c}-\epsilon l_{1}}^{t_{c}+\varepsilon l_{2}}\left[H\left(t, x\left(u^{1}\right)(t), w_{\rho}\left(u^{1}\right)\left(t_{c}\right), \Psi\left(u^{1}\right)(t)\right)\right. & \\
& \left.-H\left(t, x\left(u^{1}\right)(t), u^{1}(t), \Psi\left(u^{1}\right)(t)\right)\right] d t+o(\varepsilon) .
\end{aligned}
$$

This proves the first part of the theorem.

Now it remains to show that the second statement is true. For this, we note that

$$
\begin{aligned}
J\left(u^{1}\right)-J\left(u_{e}^{1}\right)=\int_{t_{c}-\varepsilon l_{1}}^{t+\epsilon l_{2}}\left[H \left(t, x\left(u^{1}\right)(t),\right.\right. & \left.V_{u^{1}}(t), \Psi\left(u^{1}\right)(t)\right) \\
& \left.-H\left(t, x\left(u^{1}\right)(t), u^{1}(t), \Psi\left(u^{1}\right)(t)\right)\right] d t \\
& +\int_{l_{c}-\epsilon l_{1}}^{t_{c}+\varepsilon l_{2}}\left[H\left(t, x\left(u^{1}\right)(t), w_{\rho}\left(u^{1}\right)\left(t_{c}\right), \Psi\left(u^{1}\right)(t)\right)\right. \\
& \left.-H\left(t, x\left(u^{1}\right)(t), V_{u^{1}}(t), \Psi\left(u^{1}\right)(t)\right)\right] d t+o(\varepsilon) .
\end{aligned}
$$


Note that for any $\varepsilon>0$, we can choose the integer $\rho$ such that $k \Gamma \rho<\varepsilon$. Thus the above equation reduces to

$$
\begin{aligned}
J\left(u^{1}\right)-J\left(u_{\varepsilon}^{1}\right)=\int_{t_{e}-e l_{1}}^{t_{e}+e l_{8}}\left[H \left(t, x\left(u^{1}\right)(t),\right.\right. & \left.V_{u^{1}}(t), \Psi\left(u^{1}\right)(t)\right) \\
& \left.-H\left(t, x\left(u^{1}\right)(t), u^{1}(t), \Psi\left(u^{1}\right)(t)\right)\right] d t+o(\varepsilon)
\end{aligned}
$$

for all integers $\rho$ such that $k \Gamma^{\rho}<\varepsilon$.

Now, we further note that

$$
H\left(t_{c}, x\left(u^{1}\right)\left(t_{c}\right), V_{u^{1}}\left(t_{c}\right), \Psi\left(u^{1}\right)\left(t_{c}\right)\right)>H\left(t_{c}, x\left(u^{1}\right)\left(t_{c}\right), u^{1}\left(t_{c}\right), \Psi\left(u^{1}\right)\left(t_{c}\right)\right)
$$

and the functions $H\left(\cdot, x\left(u^{1}\right)(\cdot), V_{u^{1}}(\cdot), \Psi\left(u^{1}\right)(\cdot)\right)$ and $H\left(\cdot, x\left(u^{1}\right)(\cdot), u^{1}(\cdot), \Psi\left(u^{1}\right)(\cdot)\right)$ are continuous on $\left(t_{c}-\varepsilon l_{1}, t_{c}+\varepsilon l_{2}\right)$. Thus, by an appropriate choice of $\varepsilon_{0}>0$, relation (56) implies

$$
\frac{1}{\varepsilon\left(l_{1}+l_{2}\right)} \cdot\left[J\left(u^{1}\right)-J\left(u_{s}^{1}\right)\right]>0
$$

for all $0<\varepsilon<\varepsilon_{0}$ and $\rho$ such that $k \Gamma^{\rho}<\varepsilon$.

This completes the proof.

This last theorem paves the way for the algorithm in the next section. In effect, it tells us that if we perturb a given control $u$, by replacing the original control on some interval $\Omega_{\epsilon}$ by that control which approximately maximizes

$$
H(t, x(u)(t), \cdot, \Psi(u)(t))
$$

at a point $t_{c}$ in $\Omega_{\epsilon}$, then this new control, $u_{\varepsilon}^{1}$, will improve the cost functional. This is the result that suggests a method that can be used to construct a sequence of controls $\left\{u^{\sigma}\right\}$ from any non-extremal control $u^{0}$, so that

for all positive integers $\sigma$.

$$
J\left(u^{\sigma}\right)>J\left(u^{\sigma+1}\right)
$$

\section{The algorithm}

In this section, Theorem 4.5 is used to outline the form that the algorithm should take. After an initial control $u^{0}$ and an $\varepsilon>0$ are chosen, the interval $[0, T]$ is divided up into $\left[\theta_{j}, \theta_{j+1}\right]$ for all $\theta_{j}, \theta_{j+1} \in \Theta\left(u^{0}\right)$. This is done so as to satisfy the assumptions of Theorems 4.4 and 4.5 , that $\Omega_{\varepsilon}$, the interval where the control is varied, cannot contain any of the $\theta \in \Theta\left(u^{0}\right)$, as an interior point.

Using the control $u^{0}$, the state $x\left(u^{0}\right)(t)$ is found by integrating equation (1) with the initial condition (2) forward in time and then $\psi\left(u^{0}\right)(t)$ is found by integrating 
the costate equations (4) with the final condition (5) backwards in time, both integrations being done within the sub-intervals mentioned above.

With the state and costate variables known, an approximate maximizing control $w_{\rho}\left(u^{0}\right)$ is calculated at each net point by the sub-procedure $S$. These net points are constructed in steps 6-8. In view of step 6, we note that the construction of these net points depends on the quantity $\varepsilon$. The net point that induces the largest $\Delta H$ (see step 10) is taken as the focal point around which the change of the control $u^{0}$ to $w_{p}\left(u^{0}\right)$ is contracted, if at ail, until an improvement in the cost is incurred. If no improvement is found, then $\varepsilon$ reduces to $\frac{1}{2} \varepsilon$ and the procedure begins anew. Although the control may be changed on more than one $\varepsilon$-interval, eventually we will be able only to change it at one and in this way the algorithm will in some sense converge to the structure of the perturbed control in Theorem 4.5. At this point, the theorem plays its role of ensuring that the cost functional will be improved if $\varepsilon>0$ is made sufficiently small and $w_{\rho}$, constructed by the subprocedure $S$, is such that $k \Gamma^{\rho}<\varepsilon$.

\section{Algorithm}

1. Select a piecewise constant function $u^{0}$ from $D$ and an $\varepsilon>0$.

2. Set $\alpha=0$.

3. Break up the interval $[0, T]$ into $\left[\theta_{j}, \theta_{j+1}\right]$ for all $\theta_{j}, \theta_{j+1} \in \Theta\left(u^{\alpha}\right)$, where $\Theta\left(u^{\alpha}\right)$ is as defined in Remark 3.6.

4. Calculate $x\left(u^{\alpha}\right)(\cdot)$ by solving forward in time

$$
\left\{\begin{aligned}
\dot{x}\left(u^{\alpha}\right)(t) & =\sum_{j=0}^{s} f^{j}\left(x\left(u^{\alpha}\right)\left(t-h_{j}\right), u^{\alpha}\left(t-h_{j}\right), t-h_{j}\right), \quad t \in[0, T], \\
x(t) & =\phi(t), \quad t \in\left[-h_{s}, 0\right] .
\end{aligned}\right.
$$

Note that the integration is done in each sub-interval $\left[\theta_{j}, \theta_{j+1}\right], \theta_{j}$, $\theta_{j+1} \in \Theta\left(u^{\alpha}\right)$ successively. Further, when the integration is done over the subinterval $\left[\theta_{j}, \theta_{j+1}\right]$, the initial function is taken as $x\left(u^{\alpha}\right)(t), t \in\left[\theta_{j}-h_{s}, \theta_{j}\right]$, which is obtained from the integration done over the previous sub-intervals.

5. Calculate $\psi\left(u^{\alpha}\right)(\cdot)$ by solving backwards in time

$$
\left\{\begin{array}{l}
\psi_{i}(u)(t)=-\sum_{k=0}^{n} \sum_{j=0}^{s} \frac{\partial f_{k}^{j}\left(t, x\left(u^{\alpha}\right)(t), u^{\alpha}(t)\right)}{\partial x_{i}} \psi_{k}\left(u^{\alpha}\right)\left(t+h_{j}\right), \quad i=1, \ldots, n, \\
\psi_{0}\left(u^{\alpha}\right)(t)=-1
\end{array}\right.
$$


with the final condition

$$
\begin{cases}\psi_{i}\left(u^{\alpha}\right)(t)=0 & \text { for all } t \geqslant T, \quad i=1, \ldots, n, \quad \text { and } \\ \psi_{0}\left(u^{\alpha}\right)(t)=0 & \text { for all } t>T .\end{cases}
$$

Similarly, the integration is done in each subinterval $\left[\theta_{j}, \theta_{j+1}\right], \theta_{j}, \theta_{j+1} \in \Theta\left(u^{\alpha}\right)$ successively.

6. Calculate

$$
\beta_{\gamma}=\left[\frac{\theta_{\gamma+1}-\theta_{\gamma}}{\varepsilon}\right]+1
$$

for each $\theta_{\gamma} \in \Theta\left(u^{\alpha}\right)$, where [a] means the greatest integer less than or equal to $a$. Also find $B=\sum_{\gamma=0}^{\gamma^{\prime}} \beta_{\gamma}$ where $\gamma^{\prime}$ is the largest value that $\gamma$ takes.

7. Compute $\Delta_{\gamma}$ by

8. Define

$$
\Delta_{\gamma}=\frac{\theta_{\gamma+1}-\theta_{\gamma}}{\beta_{\gamma}}, \quad \gamma=0, \ldots, \gamma^{\prime}
$$

$$
\hat{b}(\gamma, \zeta(\gamma))=\theta_{\gamma}+\zeta(\gamma) \cdot \Delta_{\gamma}
$$

where $\gamma=0,1, \ldots, \gamma^{\prime}$ and $\zeta(\gamma)=0,1, \ldots, \beta_{\gamma}-1$. Now assign $b(1)=\hat{b}(0,0)$, $b(2)=-\hat{b}(0,1), \ldots, b(B)=\hat{b}\left(\gamma^{\prime}, \beta_{\gamma^{\prime}}-1\right)$. These are denoted as $b(m), m=1, \ldots, B$.

9. Compute the $w_{\rho}\left(u^{\alpha}\right)(b(m)), m=1, \ldots, B$, by sub-procedure $S$ (see equation (50)), where $\rho$ is such that $k \Gamma^{\rho}<\varepsilon$.

10. Find the $m^{\prime}$ such that

where

$$
\Delta H\left(b\left(m^{\prime}\right)\right) \geqslant \Delta H(b(m)) \text { for all } m=1, \ldots, B,
$$

$$
\begin{aligned}
\Delta H(b(m))=H(b(m), & \left.x\left(u^{\alpha}\right)(b(m)), w_{\rho}\left(u^{\alpha}\right)(b(m)), \Psi\left(u^{\alpha}\right)(b(m))\right) \\
& -H\left(b(m), x\left(u^{\alpha}\right)(b(m)), u^{\alpha}(b(m)), \Psi\left(u^{\alpha}\right)(b(m))\right) .
\end{aligned}
$$

11. Set $k=0$ and define

$$
u^{\alpha, 0}(t)=w_{\rho}\left(u^{\alpha}\right)(b(m)), \quad t \in[b(m), b(m+1)), \quad m=1, \ldots, B .
$$

Go to step 13.

12. (i) If $\left(m^{\prime}+\left[B / 2^{k+1}\right]\right) \geqslant B$, then we set

$$
u^{\alpha, k}(t)= \begin{cases}w_{\rho}\left(u^{\alpha}\right)(b(m)), \quad t \in[b(m), b(m+1)), & \text { for } m \in\left\{B-\left[B / 2^{k}\right], \ldots, B\right\}, \\ u^{\alpha}(t) & \text { elsewhere. }\end{cases}
$$

(ii) If $\left(m^{\prime}-\left[B / 2^{k+1}\right]\right) \leqslant 0$ then we set

$$
u^{\alpha, k}(t)= \begin{cases}w_{\rho}\left(u^{\alpha}\right)(b(m)), \quad t \in[b(m), b(m+1)), & \text { for } m \in\left\{0, \ldots,\left[B / 2^{k}\right]\right\}, \\ u^{\alpha}(t) & \text { elsewhere. }\end{cases}
$$


(iii) Otherwise

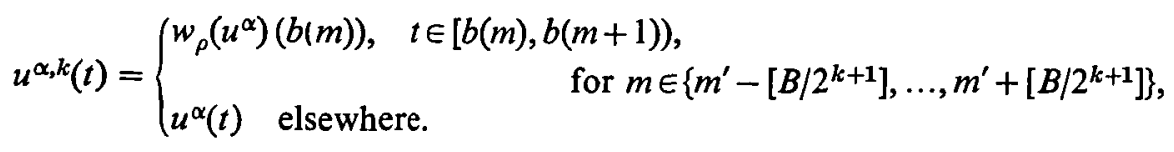

13. If $J\left(u^{\alpha, k}\right)<J\left(u^{\alpha}\right)$ (where $J$ denotes the cost functional), set

$$
u^{\alpha+1}=u^{\alpha, k}
$$

and then $\alpha=\alpha+1$ and go to step 3. Otherwise go to step 14 .

14. If $\left[B / 2^{k+1}\right]=0$ set $\varepsilon=\frac{1}{2} \varepsilon$ and go to step 6 . Otherwise set $k=k+1$ and go to step 12 .

\section{A production-inventory model}

In this section, a simple model for a production-inventory system is devised and used as an example for the computational algorithm previously proposed. In this model, various features of several models $[3,5,9]$ are incorporated to build up a picture of a firm that suffers real delayed effects in several parts of the governing processes. The first and most important delayed term arises in the difference between the managerial decision of the desired production rate and the time when it is actually implemented. The mathematical expression for this comes from Mak, Bradshaw and Porter [5],

$$
\dot{P}(t)=k\left[u_{1}\left(t-\tau_{1}\right)-P(t)\right],
$$

where $P(t)$ is the actual production rate at time $t, u_{1}(t)$ is the desired production rate at time $t$, and $\tau_{1}$ is the time delay explained above.

To distribute its goods to the outlets, it is obviously necessary to supply shipment of the products. Similarly to the first equation, the shipment rate $S(t)$ is compared to a desired shipment level, $S_{D}(t)$, which thereby determines the rate of change of $S(t)$. However, instead of taking the desired shipment rate $S_{D}(t)$ as a control variable it is calculated by an averaged difference, between the sum of unfilled orders up to time $t, U_{s}(t)$, and the sum of unfilled orders up to time $t-\tau_{3}$, $U_{s}\left(t-\tau_{3}\right)$. Therefore we have

where

$$
\dot{S}(t)=\alpha\left[S_{D}(t)-S(t)\right]
$$

$$
S_{D}(t)=\frac{\nu}{\tau_{3}}\left[U_{s}(t)-U_{s}\left(t-\tau_{3}\right)\right]
$$

With the production and shipment rates now defined, the inventory level $I$ follows immediately, that is

$$
I(t)=P(t)-S(t)
$$


The derivative of $U_{s}(t)$ will give the level of unfilled orders at time $t$,

$$
\dot{U}_{s}(t)=U(t) \text {. }
$$

In turn, the derivative of $U(t)$ will be equal to the demand rate at time $t, D(t)$, minus the shipment rate at time $t$, that is,

$$
\dot{U}(t)=D(t)-S(t) .
$$

The only factor left to be considered is the demand rate $D(t)$. In this calculation, the advertising rate $A(t)$ is assumed to play a part. Its effect on the demand rate contains a weighting factor so that the advertising done at the present will influence the change in the demand rate more than that done in the past. This is taken into account by the following equations from Connors and Teichrow [3]

$$
\dot{D}(t)=-\lambda D(t)+\gamma \int_{-\infty}^{t} A(\tau) e^{-\mu(t-\tau)} d \tau .
$$

If we now label the last term in equation (63) as the advertising level, $\Gamma(t)$, then (63) can be written as two differential equations

$$
\begin{aligned}
& \dot{D}(t)=-\lambda D(t)+\Gamma(t), \\
& \dot{\Gamma}(t)=-\mu \Gamma(t)+\gamma A(t) .
\end{aligned}
$$

If we consider how management are to decide on the advertising level, it becomes clear that there will be a time difference between the management's decision on the amount to be spent on advertising and the advertising thus generated. If an amount $u_{2}(t)$ is allocated for an advertising campaign then it will take some time for the advertising agency to devise the campaign and then, finally, present it to the public. This is represented in our model by

$$
A(t)=u_{2}\left(t-\tau_{2}\right)
$$

Equation (65) now changes to

$$
\dot{\Gamma}(t)=\mu \Gamma(t)+\gamma u_{2}\left(t-\tau_{2}\right) .
$$

All the state equations can be listed in their final form as

$$
\begin{aligned}
I(t) & =P(t)-S(t), \\
P(t) & =k\left[u_{1}\left(t-\tau_{1}\right)-P(t)\right] \\
\dot{S}(t) & =\alpha\left[\left(\nu / \tau_{3}\right)\left\{U_{s}(t)-U_{s}\left(t-\tau_{3}\right)\right\}-S(t)\right] \\
\dot{U}_{s}(t) & =U(t) \\
\dot{U}(t) & =D(t)-S(t) \\
\dot{D}(t) & =-\lambda D(t)+\Gamma(t), \\
\dot{\Gamma}(t) & =-\mu \Gamma(t)+\gamma u_{2}\left(t-\tau_{2}\right),
\end{aligned}
$$


where

$$
\begin{aligned}
& u_{1}(t)=\beta_{1}(t) \text { on }\left[-\tau_{1}, 0\right], \\
& u_{2}(t)=\beta_{2}(t) \text { on }\left[-\tau_{2}, 0\right], \\
& U_{s}(t)=g(t) \text { on }\left[-\tau_{3}, 0\right],
\end{aligned}
$$

and $I(0)=I_{0}, P(0)=P_{0}, S(0)=S_{0}, U(0)=U_{0}, D(0)=D_{0}, \Gamma(0)=\Gamma_{0}$. Both $\beta_{1}$ and $\beta_{2}$ are given piecewise continuous functions on $\left[-\tau_{1}, 0\right]$ and $\left[-\tau_{2}, 0\right]$ respectively, and $g$ is a given absolutcly continuous function on $\left[-\tau_{3}, 0\right]$.

Probably the most important aspect of any model is in deciding which factors are required to be built into the objective functional so that a realistic evaluation is presented of the give and take between cost and profit. The profit, if any, can be deduced from the shipment rate, presuming that costs and prices do not change, since the more goods the firm ships out, the more money it brings in. On the debit side, the objective functional has to take account of the extra cost of increasing the production rate from its lower limit. This lower limit signifies a production rate that does not include any overtime. Further, the objective functional must also include the cost of storing goods, the cost of advertising and, finally, the loss of sales and perhaps 'ill-will' from not being able to supply the demand, namely, a shortage cost. All this brings about the following form of $J$ which is to be maximized

$$
\begin{aligned}
J[u]=\int_{0}^{T}\left\{R_{s} S(t)\right. & -C_{P}\left[P(t)-p_{l}\right]-C_{I} I(t) e\{I(t)\} \\
& \left.-C_{U}[U(t)-I(t)] e\{U(t)-I(t)\}-C_{A} u_{2}\left(t-\tau_{2}\right)-p_{I} I(t)^{2} e\{-I(t)\}\right\} d t,
\end{aligned}
$$

where $p_{l}$ denotes the production rate with no overtime worked, $e\{\cdot\}$ is the unit step function, and $-p_{I} I(t)^{2} e\{-I(t)\}$ is a penalty for having a negative inventory, which would obviously lead to an infeasible solution.

In order to comply with assumption A(ii), we must smooth out the step function in the integrand of the objective functional (69). This is done by fitting a quadratic function in the region $-\delta \leqslant x \leqslant 0$ and another quadratic function for $0<x \leqslant \delta$, in the following manner,

$$
\hat{e}(x)= \begin{cases}0, & x<-\delta, \\ \frac{1}{2}+(x / \delta)+\frac{1}{2}\left(x^{2} / \delta^{2}\right), & -\delta \leqslant x \leqslant 0, \\ \frac{1}{2}+(x / \delta)-\frac{1}{2}\left(x^{2} / \delta^{2}\right), & 0<x \leqslant \delta, \\ 1, & x>\delta,\end{cases}
$$

where $\delta$ is "small". Clearly $\hat{e}$ is then continuously differentiable with respect to $x$ on $(-\infty, \infty)$.

Now the original optimal control problem is approximated by the one that includes the system (68) but with the objective functional altered to comply with assumption A, namely (69) with $e\{\cdot\}$ replaced by $\hat{e}\{\cdot\}$. 
Clearly, if $\delta$ is small enough in (70), the solution to the above problem shall not be very different from that of the original problem. This latter problem is the one which we shall solve.

With reference to the system (68)-(69), the Hamiltonian and the costate equations follow respectively from (6) and (4)-(5). Thus,

$$
\begin{aligned}
& H(t, x(t), u(t), \Psi(t)) \\
&=I(t) {\left[C_{U} \hat{e}\{U(t)-I(t)\}-p_{I} I(t) \hat{e}\{-I(t)\}-C_{I} \hat{e}\{I(t)\}\right] } \\
&+P(t)\left[\psi_{1}(t)-\beta \psi_{2}(t)-C_{P}\right]+S(t)\left[R_{s}-\psi_{1}(t)-\alpha \psi_{3}(t)-\psi_{5}(t)\right] \\
&+U_{s}(t)\left[\alpha \nu / \tau_{3}\left\{\psi_{3}(t)-\psi_{3}\left(t+\tau_{3}\right)\right\}\right]+U(t)\left[\psi_{4}(t)-C_{U} \hat{e}\{U(t)-I(t)\}\right] \\
&+D(t)\left[\psi_{5}(t)-\lambda \psi_{6}(t)\right]+\Gamma(t)\left[\psi_{6}(t)-\mu \psi_{7}(t)\right] \\
&+C_{P} p_{1}-C_{A} u_{2}(t) \hat{e}\left\{T-\tau_{2}-t\right\}+k \psi_{2}\left(t+\tau_{1}\right) u_{1}(t)+\gamma \psi_{7}\left(t+\tau_{2}\right) u_{2}(t) .
\end{aligned}
$$

The costate equations are as follows

$$
\begin{aligned}
& \psi_{1}(t)=C_{I} \hat{e}\{I(t)\}+2 p_{I} I(t) \hat{e}\{-I(t)\}-C_{U} \hat{e}\{U(t)-I(t)\} \\
& \psi_{2}(t)=C_{P}+k \psi_{2}(t)-\psi_{1}(t) \\
& \psi_{3}(t)=\psi_{5}(t)+\alpha \psi_{3}(t)+\psi_{1}(t)-R_{s} \\
& \psi_{4}(t)=\alpha \nu / \tau_{3}\left(\psi_{3}\left(t+\tau_{3}\right)-\psi_{3}(t)\right) \\
& \psi_{5}(t)=C_{U} \hat{e}\{U(t)-I(t)\}-\psi_{4}(t) \\
& \psi_{6}(t)=\lambda \psi_{6}(t)-\psi_{5}(t) \\
& \psi_{7}(t)=\mu \psi_{7}(t)-\psi_{6}(t)
\end{aligned}
$$

where $\psi_{i}(t)=0$ for $t \geqslant T, i=1, \ldots, 7$.

The numerical values given to the coefficients and parameters of the problem are

$$
\begin{aligned}
& R_{s}=20.0, \quad C_{P}=10.0, \quad C_{I}=1.0, \quad C_{U}=3.0, \\
& C_{A}=2.0, \quad p_{l}=1.0, \quad \alpha=2.5, \quad k=5.0 \text {, } \\
& \nu=1.0, \quad \lambda=0.05 \quad \gamma=0.016, \quad \tau_{1}=1.0 \\
& \tau_{2}=2.0, \quad \tau_{3}=1.0, \quad \mu=0.2, \quad p_{I}=1000.0 .
\end{aligned}
$$

The initial conditions for the state equations are

$$
\begin{aligned}
I(0) & =0.1, \quad P(0)=2.0, \quad S(0)=0.6, \quad U_{s}(0)=0.8, \\
U(0) & =1.0, \quad D(0)=0.8, \quad \Gamma(0)=0.0, \\
U_{s} & =0.8(t+1), \quad t \in[-1,0) .
\end{aligned}
$$


The controls are constrained to lie within the following limits

$$
\begin{aligned}
& 1.0 \leqslant u_{1} \leqslant 2.0, \\
& 1.0 \leqslant u_{2} \leqslant 2.0,
\end{aligned}
$$

and the initial controls are

$$
\begin{array}{ll}
u_{1}(t)=2.0, & t \in[-1,0), \\
u_{2}(t)=1.0, & t \in[-2,0) .
\end{array}
$$

The time interval studied was from 0 to 25 , that is, $T=25$.

The most prominent feature in the graphs is the large build-up of inventory at the beginning. This occurs because, as yet, the product is not well known to the public as can be seen from the curves representing the demand rate, $D$, and the level of unfilled orders, $U$. To overcome this, the management must spend as much on advertising as possible, hence the control $u_{2}$ moves to its maximum level.

At the beginning of the process, the control $u_{1}$ for the production rate drops to its lower level and then remains there until around $t=15$. The use of the lower production rate, while the product is still building up a market for itself, should obviously be the case, since the only thing we would achieve by using overtime would be to increase the inventory even more and, in so doing, increase costs through overtime wages and warehouse storage prices.

The deciding factor in all of these decisions is the demand rate $D$. At the beginning, it is not high enough to warrant overtime production, but this lack of demand does require the management to spend, as much as possible to get its product well known. Once the demand rate has achieved a suitable level, then the maximum amount of advertising can be dropped, and in its place goes the lower advertising level with only infrequent large advertising campaigns to keep the product in "demand". At the same time, the decrease in inventory following the increased demand has brought the inventory down to its "optimal" level, and therefore the production rate alters between its upper and lower levels to keep it there.

The other two state variables worthy of mention, the shipment rate $S$ and the level of unfilled orders $U$, follow the curve of the demand rate fairly closely, which is what one would expect.

The final stage appears when we near the end of the time interval. Here, it is obviously of no advantage to the manufacturer to have any goods left in storage or to promote its products. So both the production rate and advertising are reduced to the lower limits at such times that the inventory falls to zero at the final time.

In this way, the "best" profit of 435 is achieved. This does not mean to say that this is the best possible figure for the profit functional. Slight improvement of the 


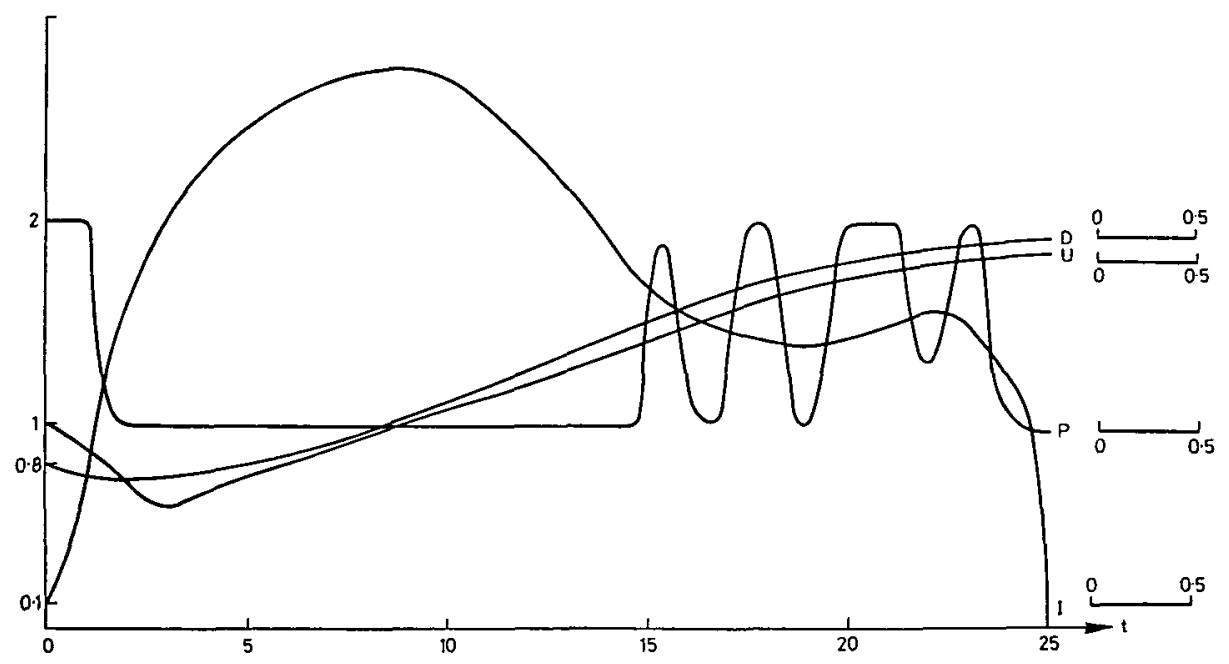

(a)

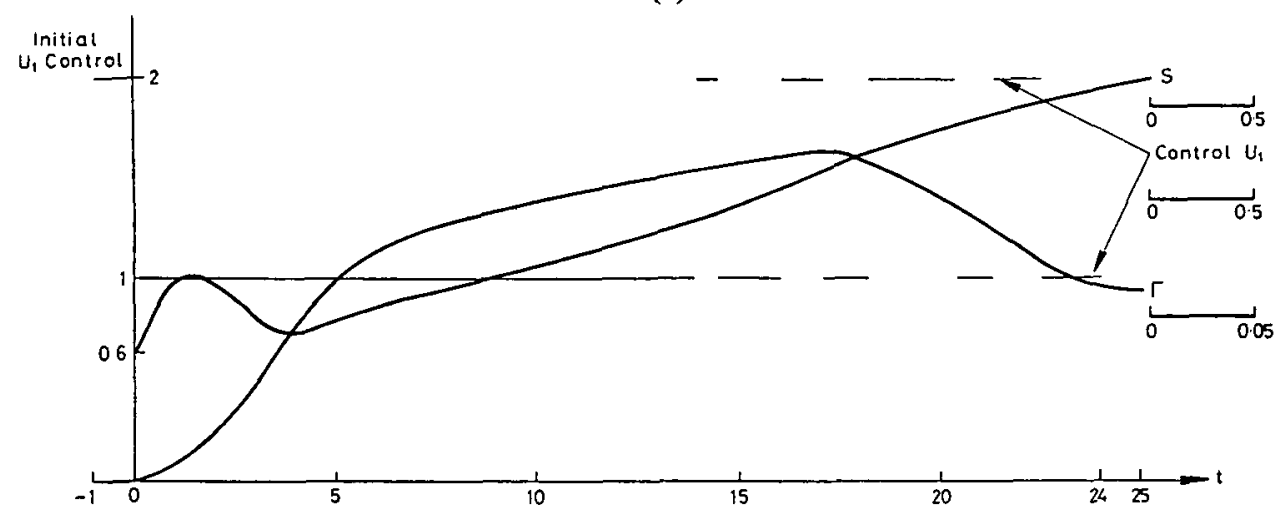

(b)

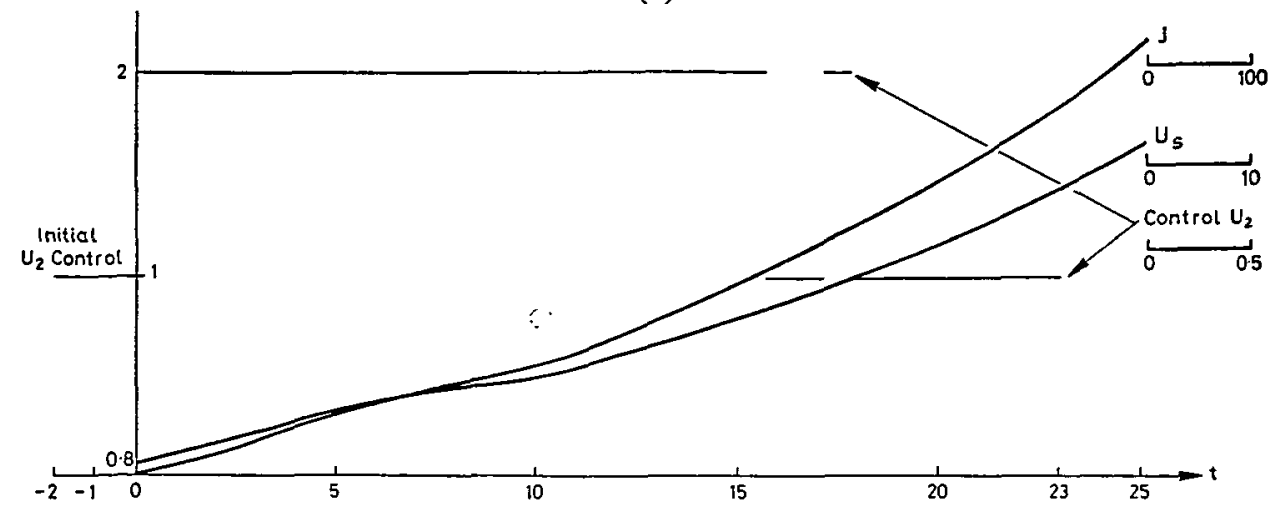

(c)

Fig. 1 (a)-(c). Illustrating the results of the production-inventory model. 
profit functional is still possible. However, it will require a lot of additional computation time. The results presented in the graphs are obtained by allowing only one halving of the initial $\varepsilon$, which was chosen to be 0.25 , as regards to step 14 of the algorithm.

Note that the controls $u_{1}$ and $u_{2}$ are only decisions made by the managers and not actual production or advertising. The production and advertising that arise from these controls occur after the decisions are made, the time differences being taken into account by the delays $\tau_{1}$ and $\tau_{2}$.

The results of the production-inventory model are illustrated in Figs. 1(a)-(c).

\section{Conclusions}

This paper has presented a computational algorithm for solving a certain type of time-delayed optimal control problem. The basis of the algorithm was given in Theorem 4.5. This theorem suggested if $u$ were any non-extremal control, then we could always construct a new control from $u$ such that the objective functional would be improved. A simple production-inventory model has been used as an example to demonstrate the effectiveness of the algorithm.

\section{Acknowledgement}

The authors wish to thank L. T. Yeo for his considerable help in the computational aspects of this paper. Further, they must also thank the referees and editor for their most helpful comments.

\section{References}

[1] L. D. Berkowitz, Optimal control theory (New York: Springer-Verlag, 1974).

[2] J. M. Blatt, "Control systems with time lags", J. Aust. Math. Soc. B 19 (1976), 478-492.

[3] M. M. Connors and D. Teichroew, Optimal control of dynamic operations research models (Scranton, Pennsylvania: International Textbook Company, 1967).

[4] H. Halkin, "Mathematical foundations of system optimization", in Topics in optimization (ed. G. Leitmann) (New York: Academic Press, 1967).

[5] K. L. Mak, A. Bradshaw and B. Porter, "Stabilizability of production-inventory systems with retarded control policies", Int. J. Systems Sc. 3 (1976), 277-288.

[6] D. Q. Mayne and E. Polak, "First-order strong variation algorithms for optimal control", J.O.T.A. 16 (1975), 277-301.

[7] E. Polak, Computational methods in optimization, a unified approach (New York: Academic Press, 1970). 
[8] K. L. Teo and L. T. Yeo, "On the computational methods of optimal control problems with applications to the decision making of a production firm" (submitted for publication).

[9] K. L. Teo, G. C. I. Lin and L. T. Yeo, "On the optimal control of a manufacturing firm", Bull. Aust. Math. Soc. 16 (1977), 111-123.

Department of Applied Mathematics

University of New South Wales

P.O. Box 1, Kensington

N.S.W. 2033

Australia 